\title{
Serine-threonine kinase ROCK2 regulates germinal center $B$ cell positioning and cholesterol biosynthesis
}

\author{
Edd Ricker, ${ }^{1,2}$ Yurii Chinenov, ${ }^{3}$ Tania Pannellini,, ${ }^{4,5}$ Danny Flores-Castro, ${ }^{1}$ Chao Ye, ${ }^{1}$ Sanjay Gupta, ${ }^{1}$ Michela Manni, ${ }^{1}$ James K. Liao, ${ }^{6}$ \\ and Alessandra B. Pernis ${ }^{1,3,7}$

\begin{abstract}
'Autoimmunity and Inflammation Program, Hospital for Special Surgery (HSS), New York, New York, USA. ²Department of Microbiology and Immunology, Weill Cornell Medicine, New York, New York, USA. ${ }^{3}$ David Z. Rosensweig Genomics Research Center, ${ }^{4}$ Research Division and ${ }^{5}$ Precision Medicine Laboratory, HSS, New York, New York, USA. ${ }^{6}$ Department of Medicine, University of Chicago, Chicago, Illinois,
\end{abstract} \\ USA. 'Department of Medicine, Weill Cornell Medicine, New York, New York, USA.
}

\begin{abstract}
Germinal center (GC) responses require B cells to respond to a dynamic set of intercellular and microenvironmental signals that instruct B cell positioning, differentiation, and metabolic reprogramming. RHO-associated coiled-coil-containing protein kinase 2 (ROCK2), a serine-threonine kinase that can be therapeutically targeted by ROCK inhibitors or statins, is a key downstream effector of RHOA CTPases. Although RHOA-mediated pathways are emerging as critical regulators of CC responses, the role of ROCK2 in B cells is unknown. Here, we found that ROCK2 was activated in response to key T cell signals like CD40 and IL-21 and that it regulated CC formation and maintenance. RNA-Seq analyses revealed that ROCK2 controlled a unique transcriptional program in CC B cells that promoted optimal CC polarization and cholesterol biosynthesis. ROCK2 regulated this program by restraining AKT activation and subsequently enhancing FOXO1 activity. ATAC-Seq (assay for transposase-accessible chromatin with high-throughput sequencing) and biochemical analyses revealed that the effects of ROCK2 on cholesterol biosynthesis were instead mediated via a novel mechanism. ROCK2 directly phosphorylated interferon regulatory factor 8 (IRF8), a crucial mediator of GC responses, and promoted its interaction with sterol regulatory elementbinding transcription factor 2 (SREBP2) at key regulatory regions controlling the expression of cholesterol biosynthetic enzymes, resulting in optimal recruitment of SREBP2 at these sites. These findings thus uncover ROCK2 as a multifaceted and therapeutically targetable regulator of CC responses.
\end{abstract}

\section{Introduction}

Efficient humoral responses require B cells to integrate signals that coordinate their positioning, differentiation, and metabolic reprogramming (1-3). Productive interactions between B cells and $\mathrm{T}$ cells are central to these responses, as $\mathrm{T}$ cells provide key signals, such as CD4OL and IL-21, that instruct B cell fate decisions and promote the formation of germinal centers (GCs) (3-5). GCs are transient anatomical structures that serve as the major sites of clonal expansion, affinity maturation, and plasma cell (PC) differentiation (1-3). The mature GC is histologically characterized by the appearance of 2 polarized microenvironments that facilitate the compartmentalization of specific functional processes (3). The GC "dark zone" (DZ) is primarily composed of rapidly dividing centroblasts and is the major site of somatic hypermutation (SHM) (3). In contrast, the GC "light zone" (LZ) is believed to be the site where affinity maturation occurs (3). The gene signature of GC B cells is controlled by an antagonistic network of transcription factors centered around BCL6 that is absolutely required for the formation and maintenance of the GC B cell program (4). Other

Conflict of interest: ABP previously received an investigator-initiated research grant from Kadmon Corporation for studies of SLE T cells.

Copyright: (5) 2020, American Society for Clinical Investigation.

Submitted: August 7, 2019; Accepted: March 24, 2020; Published: June 2, 2020

Reference information: / Clin Invest. 2020;130(7):3654-3670.

https://doi.org/10.1172/JCI132414. transcription factors also play important roles in the regulation of GC responses. In particular, interferon regulatory factor 8 (IRF8) and PU.1 control GC development through several mechanisms including the regulation of BCL6 expression (6-10) and FOXO1, a DZ-specific transcription factor that helps maintain GC architectural polarity (11-13).

In addition to interactions with $\mathrm{T}$ cells, GC $\mathrm{B}$ cells respond to microenvironmental cues that regulate key GC processes and reinforce the transcriptional networks driving $\mathrm{B}$ cell differentiation (2). B cell positioning within the GC is controlled by several GPCRs and their chemokine and lipid ligands, including CXCL13, CXCL12, S1P, 7 $\alpha, 25-\mathrm{HC}$, and S-geranylgeranyl-L-glutathione (2, 14-17). Disruption of these signaling pathways results in abnormal GC architecture, impaired B cell differentiation, and attenuated humoral responses (2). GC B cells also face increased energetic and biosynthetic demands (18), and the maintenance of GC responses requires the metabolic reprogramming of $\mathrm{B}$ cells via several metabolic sensors, in particular mechanistic target of rapamycin complex 1 (mTORC1) and glycogen synthase kinase 3 (GSK3) (19-21). GC B cells, furthermore, undergo substantial rewiring of their B cell receptor (BCR) and CD40 signaling pathways, which, in the case of the BCR, results in differential AKT phosphorylation and activity $(13,22)$.

The RHO kinases (ROCKs) ROCK1 and ROCK2 are a pair of ubiquitously expressed serine-threonine kinases that are key downstream effector molecules of RHOA (23-25). Upon RHOA 
A

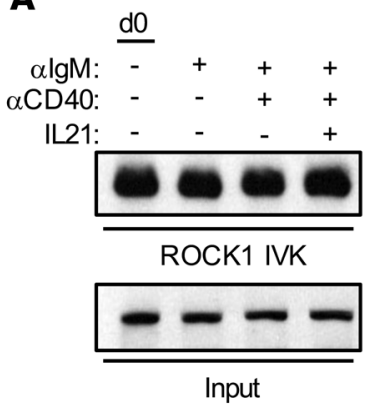

C

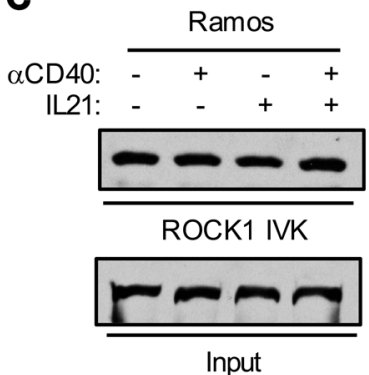

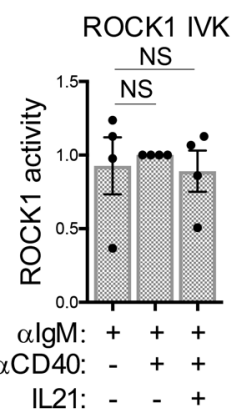

ROCK1 IVK

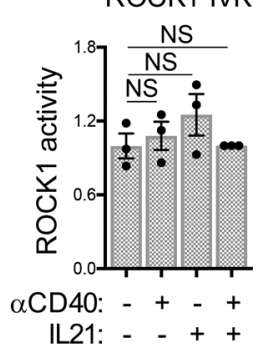

B

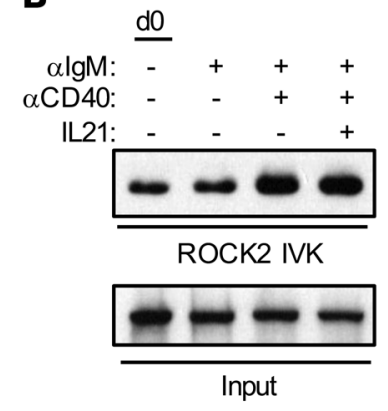

D

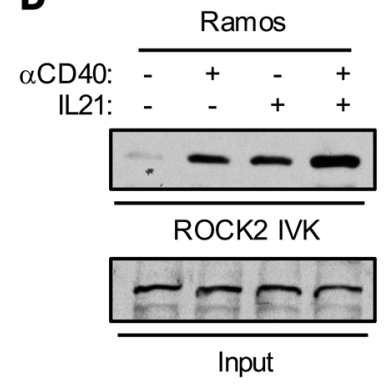

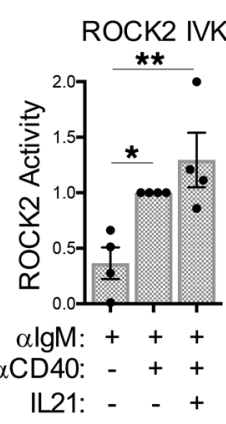

ROCK2 IVK

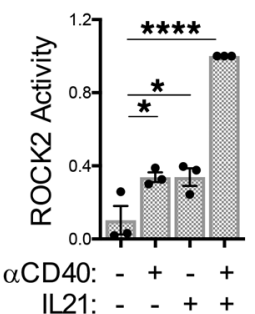

Figure 1. ROCK2 is activated by CD40 and IL-21 signals. (A and B) CD23+ B cells purified from C57BL/6 mice were collected immediately or cultured for 3 days with anti-lgM ( $\alpha$ lgM) $(5 \mu \mathrm{g} / \mathrm{mL})$, anti-CD40 $(5 \mu \mathrm{g} / \mathrm{mL})$, and/or IL-21 $(50 \mathrm{ng} / \mathrm{mL})$. ROCK1 and ROCK2 kinase activity was examined by incubating immunoprecipitated ROCK1 (A) or ROCK2 (B) from extracts with purified recombinant MYPT1 as a substrate. Phosphorylated recombinant MYPT1 ( $p$-MYPT1) was detected using an antibody against p-MYPT1. Total ROCK1 and ROCK2 input levels for each sample are shown in the lower panels. Quantifications show the ratio of p-MYPT1 to input ROCK protein expression. $n=4$. (C and D) Ramos cells were either left unstimulated or stimulated for 6 hours with anti-CD40 $(1 \mu \mathrm{g} / \mathrm{mL})$ and/or IL-21 (100 ng/mL). ROCK1 (C) and ROCK2 (D) IVK assays were performed on nuclear extracts of Ramos cells as in $\mathbf{A}$ and $\mathbf{B}$. Quantifications show the ratio of $\mathrm{p}$-MYPT1 to input ROCK protein expression. $n=3$. Data represent the mean $\pm \mathrm{SEM}$. ${ }^{*} P<0.05,{ }^{*} P<0.01$, and ${ }^{* * * *} P<0.0001$, by 1 -way ANOVA followed by Dunnett's test for multiple comparisons. do, day 0.

binding, the ROCKs become activated and function as central coordinators of a tissue response to stress and injury, largely due to their ability to regulate cytoskeletal rearrangements (23-25). In line with these findings, dysregulation of ROCK activity in nonhematopoietic cells has been linked to cardiovascular- and metabolism-related diseases, and a decrease in ROCK activity has been implicated in the antiinflammatory effects of statins (25). The ROCKs are activated in response to various physiological signals including those mediated by the G12/13 subfamily of GPCRs, like S1PR2, which regulates the confinement of GC B cells and whose dysregulation has been linked to $\mathrm{B}$ cell lymphomagenesis $(26,27)$. Although the ROCKs share a high degree of homology in their kinase domain and can target similar substrates in vitro, they display more limited similarity in the remainder of the molecule, suggesting that ROCK1 and ROCK2 can mediate unique sets of functions (23-25). Indeed, in T cells, ROCK2 has been shown to promote the differentiation of Th17 and $\mathrm{T}$ follicular helper (Tfh) cells because of its ability to regulate the activity and expression of IRF4 and STAT3 (28-30). Whether ROCK2 has specific roles in B cell differentiation is, however, not known.

In this study, we specifically examined the regulation and role of ROCK2 in B cells. We found that ROCK2 was selectively activated in $B$ cells in response to T cell-dependent (TD) signals and that B cell-specific deletion of ROCK2 impaired GC formation and maintenance, resulting in defective humoral responses. Through genome-wide sequencing approaches, we demonstrated that ROCK2 regulated a unique transcriptional program in GC B cells, which included both inhibitory and activating effects. In particular, ROCK2 inhibited AKT activation and promoted the transcriptional activity of FOXO1. In line with these findings, the lack of ROCK2 was associated with defective GC polarization. Interestingly, ROCK2 induced the expression of key enzymes in the mevalonate pathway by controlling the activity of sterol regulatory element-binding transcription factor 2 (SREBP2), the master regulator of cholesterol biosynthesis. These studies thus identify ROCK2 as a key regulator of the positioning and metabolic reprogramming of GC B cells.

\section{Results}

ROCK 2 is activated in response to CD4O and IL-21 signals. Although upstream components of the RHOA pathway have recently emerged as important regulators of GC responses $(16,26)$, the role of key downstream RHOA effectors like the ROCKs in GC B cells is largely unknown. To assess the involvement of the ROCKs in this compartment, we first used in vitro systems to evaluate whether ROCK activity could be regulated by key T cell-derived signals, such as those provided by CD40 engagement and IL-21, which are known to promote GC formation (3-5). Purified $\mathrm{CD}^{2} 3^{+}$follicular $\mathrm{B}$ cells (FoBs) from C57BL/6 mice were stimulated with different combinations of anti-IgM and anti-CD40 antibodies and IL-21. As reported (31), stimulation of $\mathrm{CD} 23^{+} \mathrm{B}$ cells with IL-21 in the presence of BCR and CD4O engagement induced Bcl6 and Aicda and repressed Irf4 expression (Supplemental Figure 1A; supplemental material available online with this article; https://doi.org/10.1172/ 
A

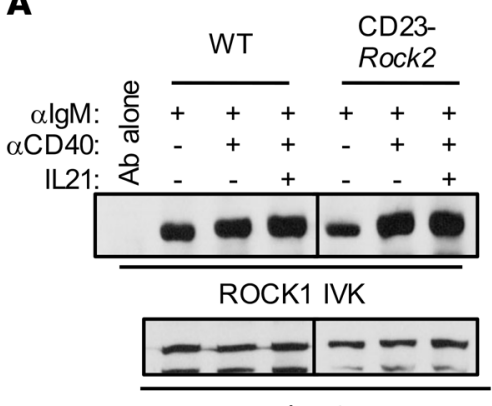

Input

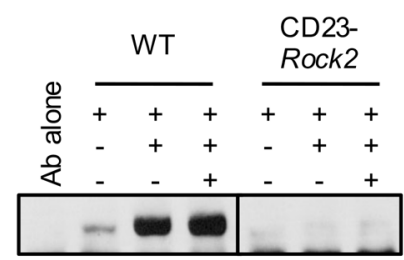

ROCK2 IVK

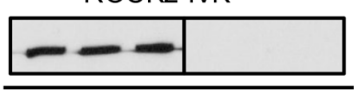

Input
B

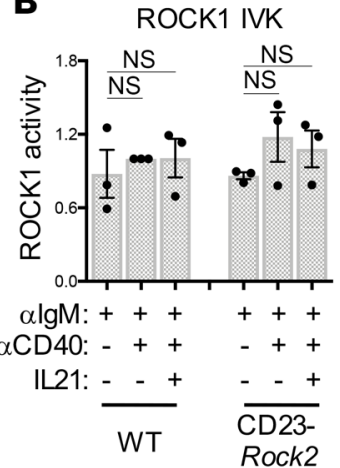

C

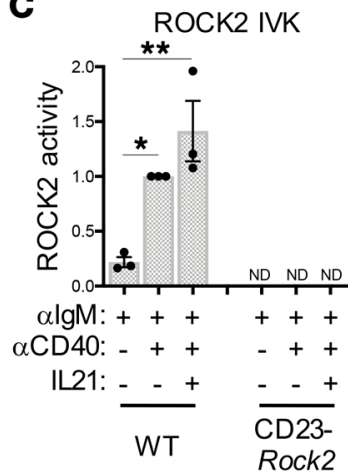

D

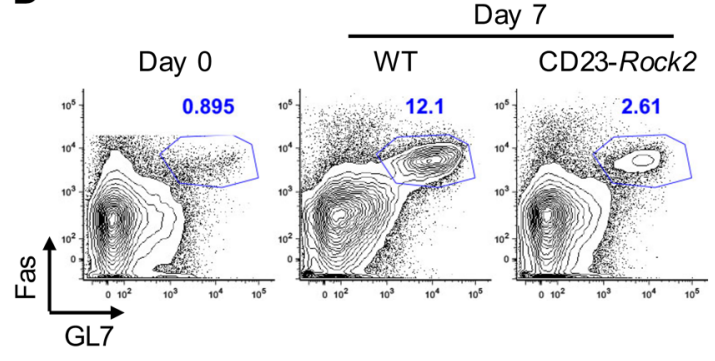

$\mathbf{F}$

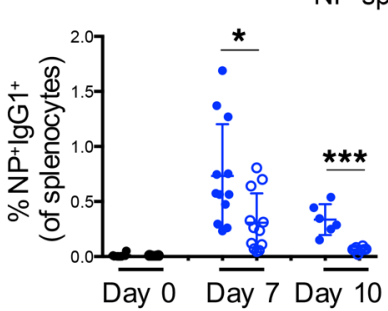

NP-specific $B$ cells

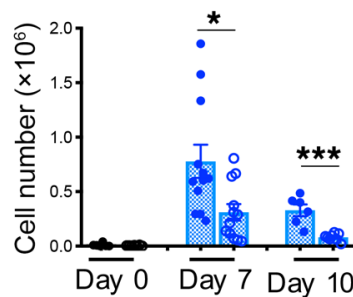

H

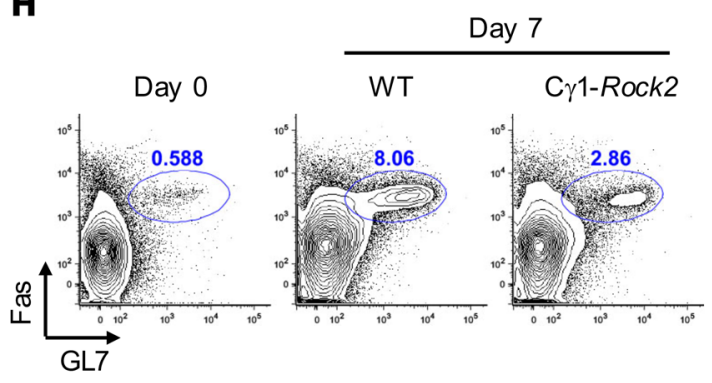

J

NP-specific B cells
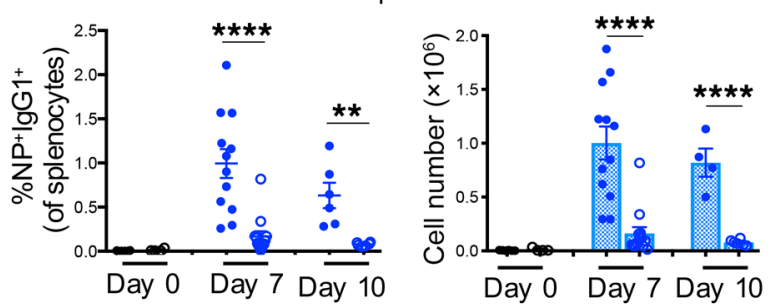

$\mathbf{E}$
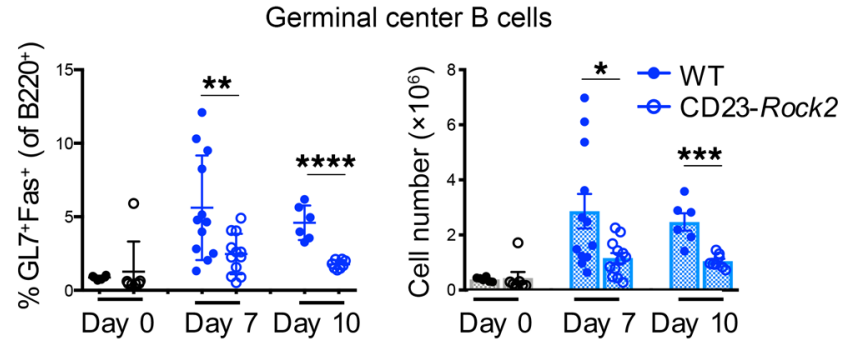

G
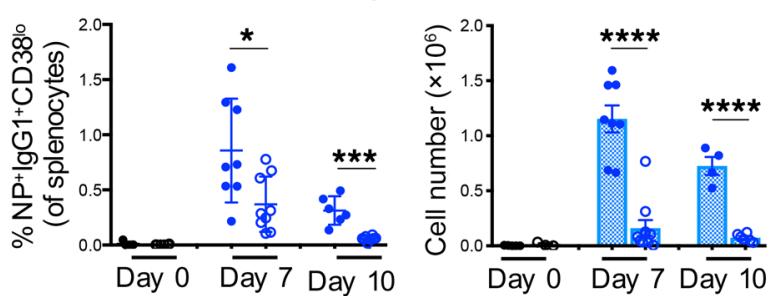

I

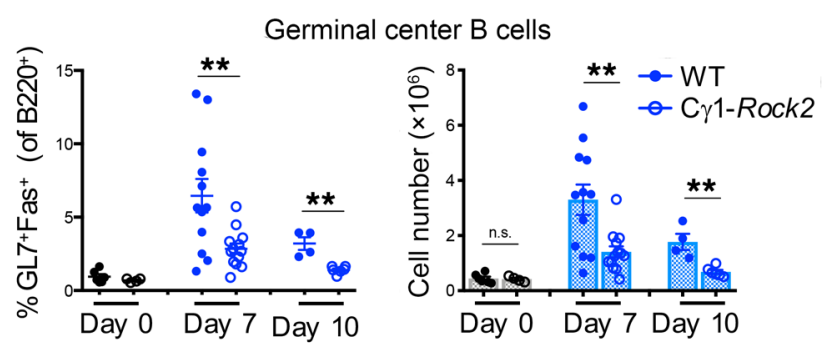

$\mathbf{K}$

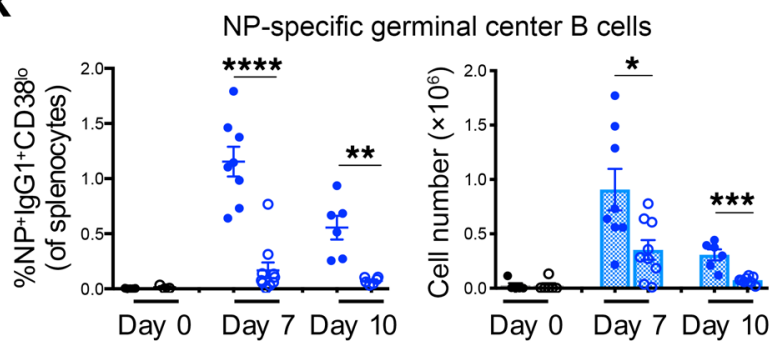


Figure 2. ROCK2 deficiency leads to impaired GC responses. (A-C) ROCK1 and ROCK2 IVK assays (A) were performed on extracts from $C D 23^{+}$cultures from Rock $\mathrm{fl}^{\prime / f l}$ (WT) or CD23-Cre Rock2 $2^{f / f l}$ (CD23-Rock2) mice as in Figure 1, $A$ and B. Kinase activity was quantified for ROCK1 (B) and ROCK2 (C) as the ratio of p-MYPT1 to input ROCK protein. $n=3$. Data represent the mean \pm SEM. $P$ values were determined by 1-way ANOVA followed by Dunnett's test for multiple comparisons. Lanes in $\mathbf{A}$ were run on the same gel but were noncontiguous. (D-G) WT and CD23-Rock2 mice were immunized with $100 \mu \mathrm{g}$ NP-CGG for 7 to 10 days. Representative FACS plots (D) and pooled quantifications of total GC B cells (E) (B220+Fas $\left.{ }^{+} \mathrm{CL} 7^{+}\right)$, total NP-specific B cells (F) (B220+|gM-lgD-Gr1-NP+|gG1+), and NP-specific GC B

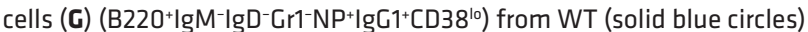
and CD23-Rock2 (open blue circles) mice. (H-K) WT and Cy1-Cre Rock $2^{f l}$ ${ }^{f l}(\mathbf{C} \gamma 1-$ Rock2) mice were immunized as in D-G. Representative FACS plots (H) and pooled quantifications of total GC B cells (I), total NP-specific B cells (J), and NP-specific GC B cells (K) from WT (solid blue) or C $\gamma 1-$ Rock2 (open blue) mice. $n>6$. Data are from at least 2 independent experiments per time point and represent the mean $\pm \mathrm{SEM} .{ }^{*} P<0.05,{ }^{* *} P<0.01,{ }^{* *} P$ $<0.001$, and ${ }^{* * *} P<0.0001$, by unpaired 2 -tailed $t$ test.

JCI132414DS1). We then performed in vitro kinase (IVK) assays to assess ROCK1- and ROCK2-specific activity in response to these signals (Figure 1, A and B). We found that ROCK1 was highly activated in B cells, irrespective of stimulation with anti-IgM, anti-CD40, or IL-21 (Figure 1A and Supplemental Figure 1B). In contrast,we detected only low levels of ROCK2 activity at baseline or following anti-IgM stimulation alone (Figure 1B). However, ROCK2 activation was strongly induced following CD4O stimulation and remained high in the presence of IL-21 (Figure 1B and Supplemental Figure 1B). Consistent with these in vitro findings, the phosphorylation of ezrin/radixin/moesin (p-ERM) proteins, which are classic ROCK targets, was increased in GC B cells and plasmablasts (PBs)/PCs from immunized mice (Supplemental Figure 1C). RHOA activity matched the ROCK1 activation pattern (Supplemental Figure 1, D and E). We found that the activity of the 2 ROCK isoforms was differentially regulated during B cell activation, with upregulation of ROCK2 activity being observed primarily following CD40 engagement.

Since stimulation of murine B cells with anti-CD 40 and IL-21 activated ROCK2, we next asked whether ROCK2 activity is also regulated by these signals in human B cells. To address this, we used a GC-derived Burkitt lymphoma cell line (Ramos) that has been previously used to study the signals driving GC exit (32, 33). ROCK1 activity was high in Ramos cells at baseline and was unaffected by stimulation with anti-CD40 or IL-21 (Figure 1C). ROCK2 activity was again low at baseline but could be robustly induced upon either CD40 engagement or IL-21 stimulation (Figure 1D). Combination of these signals further enhanced ROCK2 activity (Figure 1D). Stimulation of Ramos cells with anti-CD40 and IL-4 also promoted ROCK2 activity, suggesting that various $\mathrm{T}$ cell-derived signals known to promote humoral responses may contribute to ROCK2 activation (Supplemental Figure 1, F and G). Thus, whereas ROCK1 was constitutively activated, ROCK2 activation was controlled by key $\mathrm{T}$ cell-derived signals known to control GC B cell responses.

ROCK2 deficiency leads to impaired GC responses. The selective activation of ROCK2 in response to T cell-dependent signals raised the possibility that ROCK2 could play a functional role in regulating GC responses. To evaluate this possibility, Rock $2^{\text {f/fll }}$ mice were crossed with mice expressing Cre under the control of the Fcer2a promoter (CD23-Cre). CD23-Cre Rock2 $2^{\text {flfl }}$ (termed CD23-Rock2) mice allow for the selective deletion of Rock2 in mature B cell populations (34). Rock $2^{f / f l}$ (termed WT) mice served as controls. We confirmed Rock2 deletion in B cells purified from CD23-Rock2 mice (Supplemental Figure 2, A and B). Rock1 expression was comparable in WT and CD23-Rock2 mice (Supplemental Figure 2, A and B). We then performed IVK assays in CD23-Rock2 B cells following stimulation with anti-IgM, anti-CD40, and IL-21 (Figure 2, A-C). We found no detectable ROCK2 activity in CD23-Rock2 B cells, whereas ROCK1 activity was comparable between CD23-Rock2 B cells and WT B cells (Figure 2, A-C). Therefore, the absence of ROCK2 in B cells did not lead to enhanced ROCK1 activation.

We next examined whether B cell development was altered in the absence of ROCK2. No abnormalities in the development of bone marrow, blood, or splenic B cell populations (35) were observed in CD23-Rock2 mice (Supplemental Figure 2, C-H). Baseline levels of serum Igs were also comparable between WT and CD23-Rock2 mice (Supplemental Figure 2I). The absence of ROCK2, therefore, did not alter mature B cell development.

To directly examine the contribution of ROCK2 to GC responses, we next immunized WT and CD23-Rock2 mice with a TD antigen, 4-hydroxy-3-nitrophenol-acetyl (NP) conjugated to chicken gamma globulin (CGG). Compared with WT mice, the frequencies and numbers of GC B cells $\left(\mathrm{B}_{2} 2 \mathrm{O}^{+} \mathrm{GL}^{+} \mathrm{Fas}^{+}\right)$on day 7 and day 10 after immunization were greatly reduced in the spleens of CD23-Rock2 mice (Figure 2, D and E). We also assessed antigen-specific $\mathrm{B}$ cell responses by monitoring the binding of fluorescently labeled NP. A significant population of WT B cells bound NP by day 7 (Figure 2F and Supplemental Figure 3A). The frequency and total numbers of NP-specific B cells $\left(\mathrm{B} 22 \mathrm{O}^{+}\right.$ $\left.\operatorname{IgM}^{-} \operatorname{IgD}{ }^{-} \mathrm{Gr}^{-} \mathrm{NP}^{+} \operatorname{IgG} 1^{+}\right)$in the spleens of CD23-Rock2 mice were greatly reduced compared with WT mice, an effect that we also observed on day 10 after immunization (Figure 2F and Supplemental Figure 3A). Consistent with the decreases in total NPspecific $B$ cells, we also observed a marked reduction in NPspecific GC B cells $\left(\mathrm{CD} 38^{\circ}\right)$ in CD23-Rock2 mice compared with WT mice on days 7 and 10 after immunization (Figure 2G and Supplemental Figure 3A). B cell-specific ROCK2 was thus required for optimal GC B cell responses.

To assess whether ROCK2 also plays a role in the maintenance of GC B cell responses, we crossed Rock $2^{f / /}$ mice with mice expressing $\mathrm{C} \gamma 1-\mathrm{Cr}$, which deletes during the early stages of the GC reaction $(36,37)$. Rock $2^{f / f l}$ (WT) mice again served as a control for these experiments. Similar to what we observed in CD23-Rock2 mice, C $\gamma 1$-Cre Rock $2^{f / f l}$ (termed C $\gamma 1$-Rock2) mice had markedly reduced GC B cells compared with WT mice (Figure 2, H and I). GC B cells from $\mathrm{C} \gamma 1-$ Rock2 mice also exhibited significantly reduced class switching to IgG1 and were less proliferative than were WT GC B cells (Supplemental Figure 3, B and C). Antigen-specific responses were also greatly decreased in C $\gamma 1-$ Rock2 mice compared with responses in WT mice (Figure 2, J and K, and Supplemental Figure 3D). Thus, the lack of ROCK2 in B cells resulted in defective formation and/or maintenance of GC B cell responses.

Loss of ROCK 2 impairs PC formation and humoral responses. Given the impact of B cell ROCK2 on GC formation and mainte- 
A

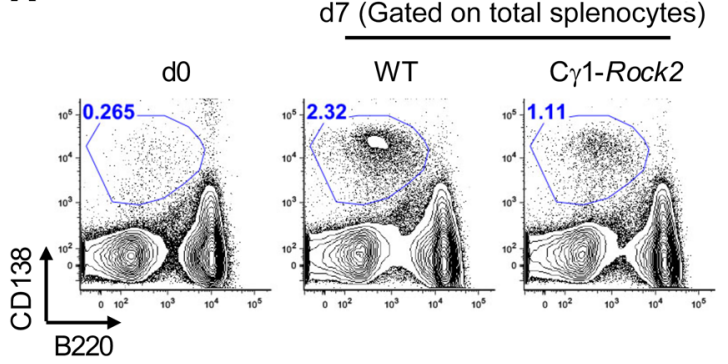

C

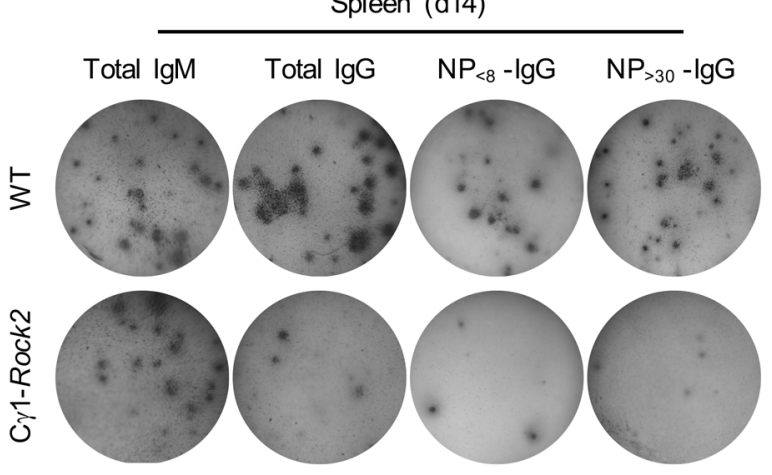

E

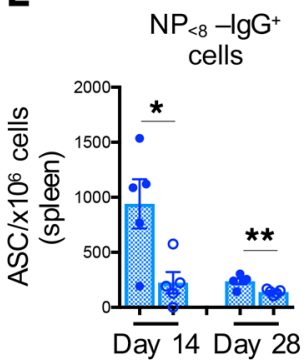

$\mathrm{NP}_{>30}-\lg \mathrm{G}^{+}$ cells

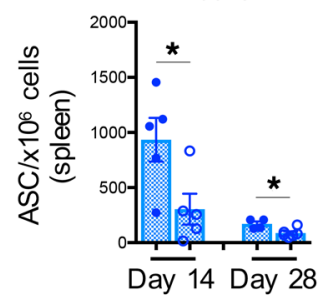

$\mathbf{F}$
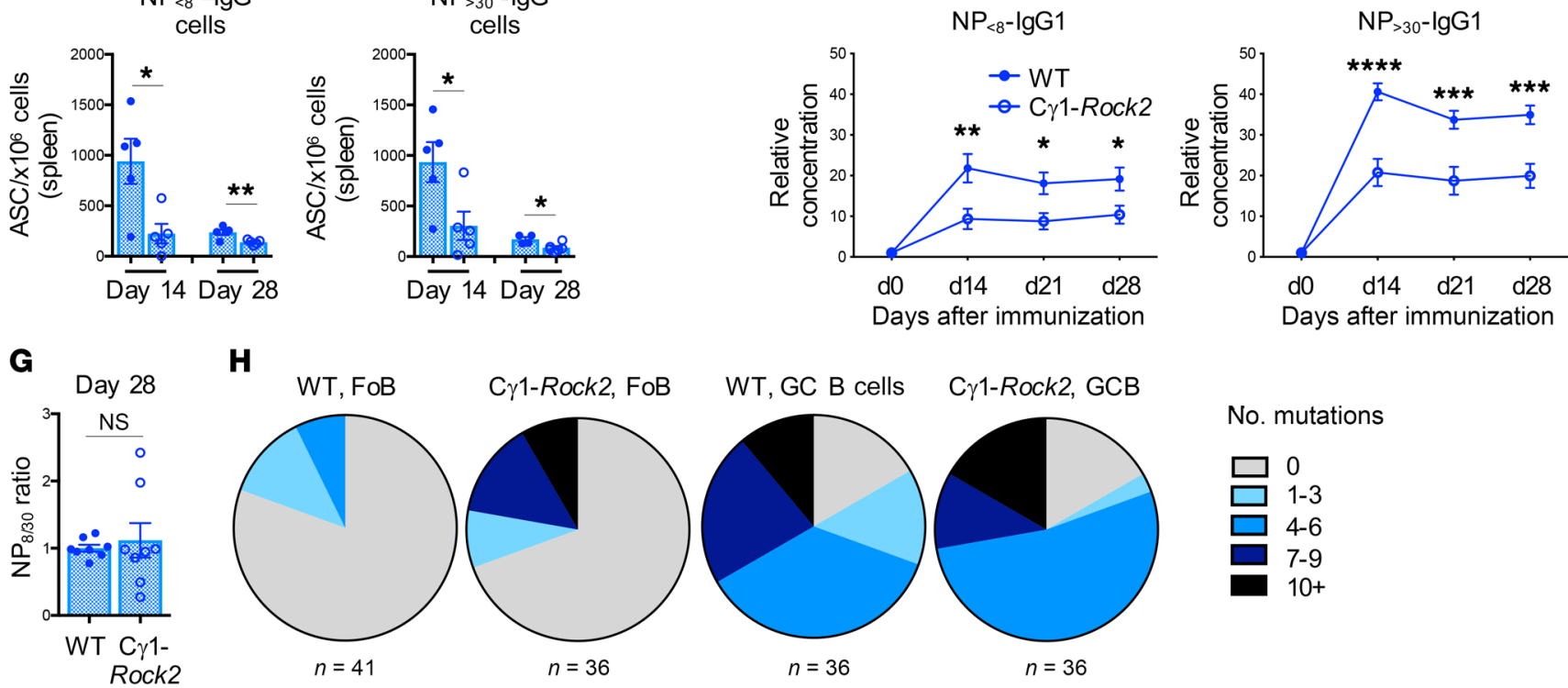

H
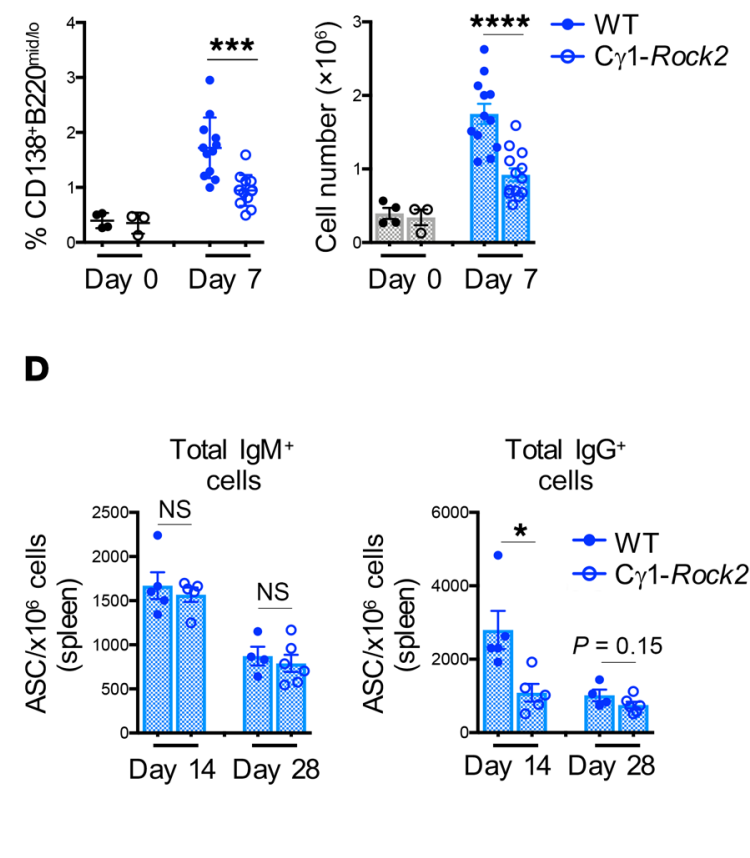

D

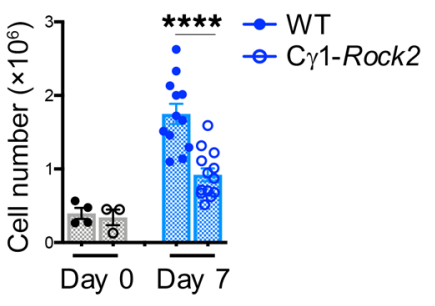

Day 14 Day 28
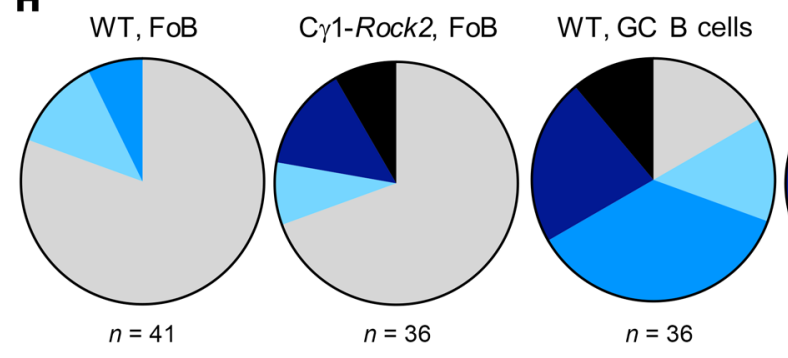

C $\gamma 1-R o c k 2, G C B$

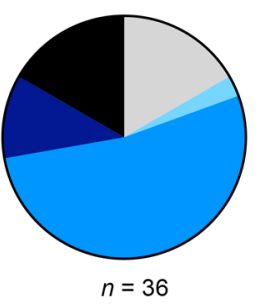

No. mutations

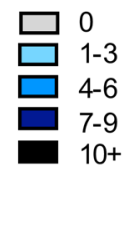

I

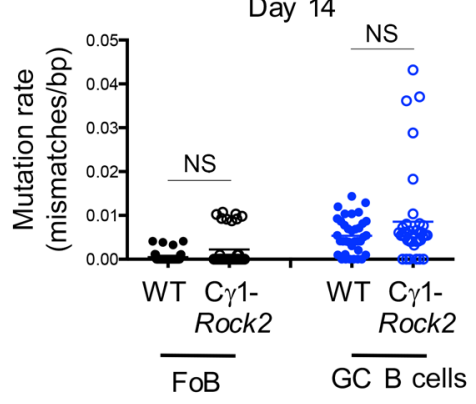


Figure 3. Loss of ROCK2 impairs PC formation and humoral responses. Rock $2^{f / f l}$ (WT) or C $\gamma 1$-Rock2 mice were immunized with $100 \mu \mathrm{g}$ NP-CGG for 7 to 28 days. (A and B) Representative FACS plots (A) and pooled quantifications (B) of PB/PCs (B220 $\left.{ }^{\circ} \mathrm{CD} 138^{+}\right)$from WT (solid blue circles) or $\mathrm{C} \gamma 1-$ Rock2 (open blue circles) mice on day 7 after immunization. $n>12$. Data are from 4 independent experiments and represent the mean \pm SEM. ${ }^{* *} P$ $<0.001$ and ${ }^{* * *} P<0.0001$, by unpaired, 2 -tailed $t$ test. (C-E) Representative ELISPOT images (C) and quantifications of total (D) and NP-specific (E) ASCs in spleens from the indicated mice on day 14 and day 28 after immunization. $n>4$. Data are from 2 independent experiments per time point and represent the mean $\pm \mathrm{SEM} .{ }^{*} P<0.05$ and $P=0.15$, by unpaired, 2-tailed $t$ test. (F) Pooled ELISA analysis of $\mathrm{NP}_{<8}$-specific and $\mathrm{NP}_{>30}$-specific IgG1 from serum of the indicated mice from days $0-28$ after immunization. $n=8$. Data were pooled from 2 independent experiments and represent the mean $\pm \mathrm{SEM}$. ${ }^{*} P<0.05$, ${ }^{*} P<0.01$, ${ }^{* *} P<0.001$, and ${ }^{* * *} P<0.0001$, by 2-way ANOVA followed by Holm-Šidák's test for multiple comparisons. (G) Ratio of $\mathrm{NP}_{<8}$-specific IgG1 to $\mathrm{NP}_{>30}$-specific lgG1 titers in serum from the indicated mice on day 28 after immunization. $n=8$. Data are from 2 independent experiments and represent the mean \pm SEM. Statistical significance was determined by unpaired, 2-tailed $t$ test. ( $\mathbf{H}$ and $\mathbf{I}$ ) Pie charts (H) and plots (I) showing the mutation frequency of the 470-bp J 4 region in sorted FoBs (B220+CL7-CD38 ${ }^{\text {hi }}{ }^{+}$D23 $)$and GC B cells (B22O+ $\left.{ }^{+} L 7^{+} C D 38^{10}\right)$ on day 14 after immunization. $n>36$ clones from 4 mice per genotype. Data were pooled from 2 independent experiments and represent the mean \pm SEM. Statistical significance was determined by unpaired, 2-tailed $t$ test.

nance, we next examined whether expression of ROCK 2 in B cells also contributed to $\mathrm{PB} / \mathrm{PC}$ formation. Compared with WT mice, the frequencies and numbers of splenic PBs/PCs $\left(\mathrm{B} 22 \mathrm{O}^{\mathrm{lo}} \mathrm{CD} 138^{+}\right)$ from $\mathrm{C} \gamma 1$-Rock2 mice on day 7 after immunization were significantly decreased (Figure 3, A and B). We also assessed PB/PC differentiation at later time points by enzyme-linked immunospot (ELISPOT) assay (Figure 3C and Supplemental Figure 4A). Compared with WT mice, the number of splenic and bone marrow $\mathrm{IgG}^{+}$antibody-secreting cells (ASCs) in C $\gamma 1-$ Rock2 mice was decreased on day 14 after immunization, whereas the number of $\mathrm{IgM}^{+}$ASCs was not affected (Figure 3, C and D, and Supplemental Figure 4, A and B). We also observed a significant reduction in the frequency of total $\left(\mathrm{NP}_{>30}\right)$ and high-affinity $\left(\mathrm{NP}_{<8}\right.$-reactive) antigen-specific $\mathrm{IgG}^{+}$ASCs in the spleens and bone marrow of immunized C 1 1-Rock2 mice compared with WT mice (Figure 3, C and E, and Supplemental Figure 4, A and B), which was accompanied by a significant reduction in both $\mathrm{NP}_{>30}-$ and $\mathrm{NP}_{<8}-$ reactive IgG1 titers (Figure 3, F and $\mathrm{G}$ ). $\mathrm{NP}_{>30}$-reactive IgM titers in C $\gamma 1$-Rock2 mice were only transiently decreased (Supplemental Figure 4C). According to these observations, the absence of ROCK2 in B cells affected both low- and high-affinity humoral responses to a similar extent.

To further assess whether ROCK2 is necessary for SHM, we sorted GC B cells from immunized WT or C $\gamma 1-$ Rock2 mice and sequenced a portion of the $\mathrm{J}_{\mathrm{H}} 4$ intron of the heavy chain variable region. The mutation frequency in this intronic region provides a surrogate measurement of activation-induced cytidine deaminase (AID) activity and SHM (38). As a control, we also sorted and sequenced the $\mathrm{J}_{\mathrm{H}} 4$ region of FoBs from WT and C $\gamma 1$-Rock 2 mice. As expected, only a few mutations were found in the FoBs from WT and C $11-R o c k 2$ mice, whereas several mutations were observed in GC B cells (Figure 3, H and I). We detected no significant difference in the mutation rate between WT and C $\gamma 1-$ Rock2 GC B cells (Figure $3 \mathrm{I})$. These data suggest that ROCK2 is not required for SHM.
ROCK2 regulates a distinctive transcriptional program in $G C B$ cells. To gain insights into the mechanisms by which ROCK2 promotes the formation and maintenance of GC B cells, we next sorted B cells from immunized mice and used RNA-Seq to compare the transcriptomes of WT and CD23-Rock2 GC B cells, using the corresponding FoBs as controls. We first confirmed the deletion of Rock2 in sorted GC B cells from CD23-Rock2 mice (Figure 4A). RNA-Seq analyses revealed that only 24 genes were differentially expressed in CD23-Rock2 FoBs compared with expression in WT FoBs $(P<0.01)$ (Figure 4B and Supplemental Table 1). Among these genes, we found that ROCK2 promoted the expression of activation-induced cytidine deaminase ( $\mathrm{Aicda}$ ) and several genes implicated in RHOA-mediated signaling pathways, including sphingosine-1-phosphate receptor 2 (S1pr2) and germinal centerassociated signaling and motility (Gcsam), the gene encoding HGAL (refs. 16, 39 and Figure 4B).

In contrast to the small number of genes differentially expressed by the FoBs from CD23-Rock2 and WT mice, a total of 284 genes were either upregulated (269 genes) or downregulated (15 genes) in CD23-Rock2 GC B cells compared with WT GC B cell genes (Figure 4C). GC B cells from WT and CD23-Rock2 mice expressed similar transcript levels of GC markers such as Bcl6, Ezh2, Bach2, Fas, and Ung, which, as expected, were higher than the transcript levels detected in FoBs (Supplemental Figure $5 A)$. FACS analysis further confirmed that the expression of key GC markers such as BCL6 and ephrin-B1 was similar in WT and CD23-Rock2 GC B cells (Supplemental Figure 5, B and C). Gene set enrichment analysis (GSEA) indicated that among the top enriched pathways (FDR $Q<0.05$ ) in CD23-Rock2 GC B cells relative to WT GC B cells were gene sets related to GPCR signaling receptor expression and activity (Figure 4, D and E, and Supplemental Figure 5D). In CD23-Rock2 GC B cells, we used real-time quantitative PCR (RT-qPCR) to confirm the upregulation of several target genes including C-C motif chemokine receptor 6 (Ccr6), Ccr7, G protein-coupled receptor 183 (Gpr183, encoding EBI2), integrin subunit $\alpha \mathrm{M}$ (Itgam), and IL-9 receptor (Il9r), known to play important roles in the localization of B cells $(2,15,17,40)$ (Figure 4F). Increased expression of a subset of these targets was also corroborated by flow cytometry of GC B cells from C $\gamma 1-R o c k 2$ mice (Supplemental Figure 5, E and F). The effect of ROCK2 on chemokine receptor expression was selective, as CXCR5 surface levels were unaffected in C $\gamma 1$-Rock2 GC B cells compared with expression in WT GC B cells (Supplemental Figure 5G). Thus, ROCK2 controlled the expression of key receptors, such as Gpr183/ $\mathrm{EBI}$, that are known to regulate the positioning of GC B cells.

To further evaluate the effects of ROCK2 deficiency on GC B cell localization, we performed an immunohistochemical analysis of the spleens of CD23-Rock2 mice. No gross abnormalities in the overall architecture of the spleens of immunized CD23-Rock2 mice were observed (Figure 4G). CD23-Rock2 mice, however, had significantly smaller GCs $\left(\mathrm{B} 220^{+} \mathrm{PNA}^{+}\right)$on day 7 after immunization compared with WT mice (Figure $4 \mathrm{H}$ ). Since a lack of ARHGEF1, an upstream regulator of ROCK activation, leads to the hematogenous spread of GC B cells (26), we also assessed whether an absence of ROCK 2 in $\mathrm{B}$ cells resulted in an increased number of GC B cells in the blood. No GC B cells were observed in the blood of young immunized CD23-Rock2 mice (Supplemental Fig- 
A

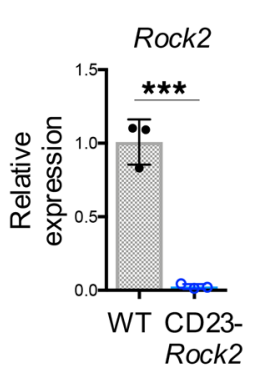

B

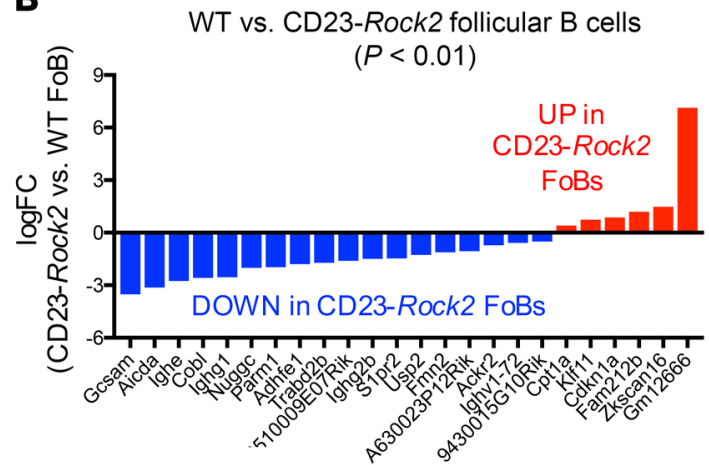

C

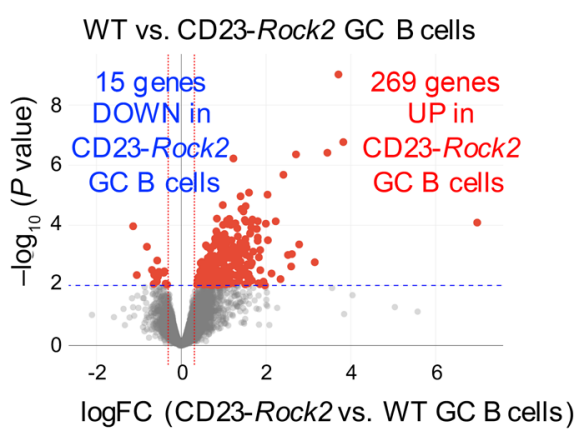

E

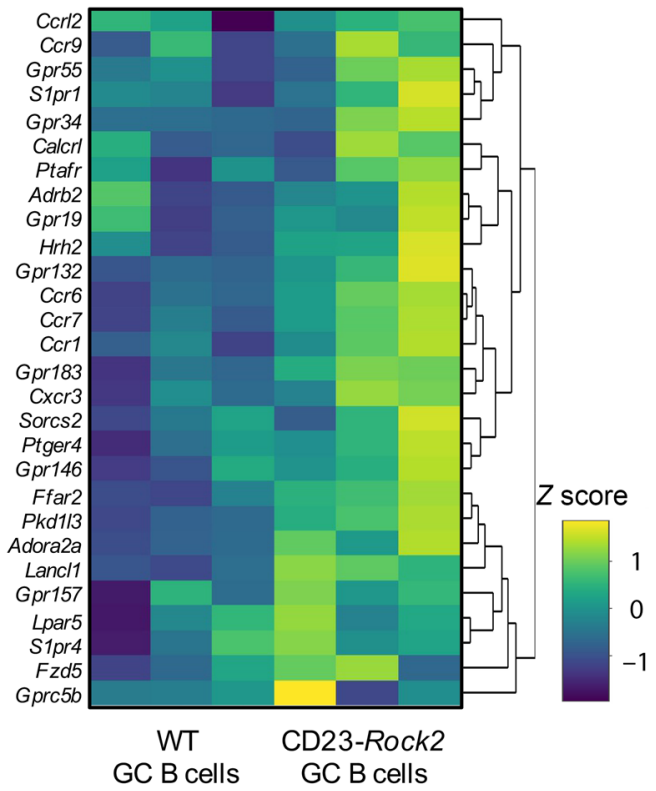

$\mathbf{F}$
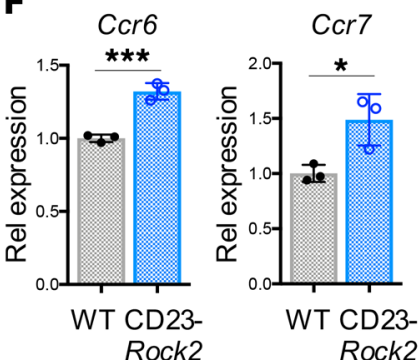

Rock2

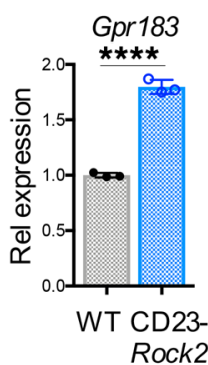

Rock2

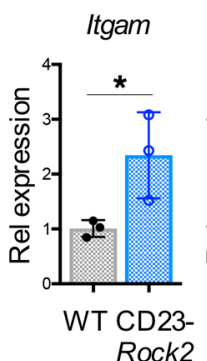

Rock2

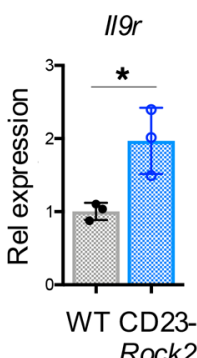

Rock2
G

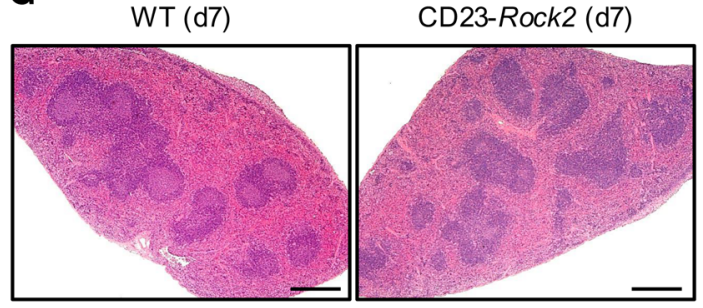

H WT

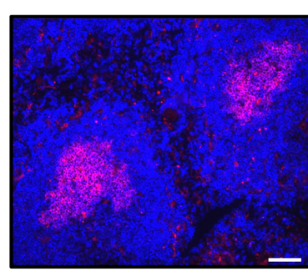

B220 PNA
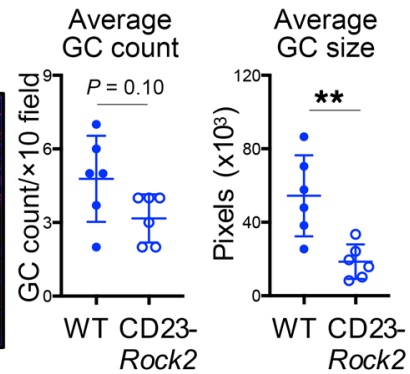

Figure 4. ROCK2 regulates a distinctive transcriptional program in GC B cells. WT or CD23-Rock2 mice were immunized with $100 \mu \mathrm{g}$ NP-CGG for 7 days, and FoBs (B220+CL7-CD38 ${ }^{\text {hi } C D 23^{+}}$) and GC B cells (B220 ${ }^{+} \mathrm{CL7} 7^{+} \mathrm{CD} 38^{\circ}$ ) were sorted for RNA-Seq analyses. (A) Representative RT-qPCR analysis of Rock2 in sorted FoBs and GC B cells from the indicated mice. $n=4$. Data are from technical triplicates and are representative of 4 independent experiments. Data represent the mean $\pm \mathrm{SD}$. ${ }^{* *} P<0.001$, by unpaired, 2-tailed $t$ test. (B) Plot shows the log-transformed FC (logFC) values for genes differentially expressed (unadjusted $P<0.01$ ) between WT and CD23-Rock2 FoBs. (C) Volcano plot shows the genes differentially expressed (unadjusted $P<$ 0.01) between WT and CD23-Rock2 GC B cells. 46. The unadjusted $P$ values in B and C were determined using edgeR3.24.3 in R. (D) Plot shows the top enriched gene sets by GSEA that were upregulated in CD23-Rock2 GC B cells. Dotted line indicates the significance cutoff at a FDR of $Q=0.05$. GO, Gene Ontology. (E) Heatmap of the scaled expression of genes enriching the GPCR_Activity gene set in CD23-Rock2 GC B cells. (F) Representative RT-qPCR analyses of the indicated genes in sorted GC B cells. $n=4$. Data show technical triplicates and are representative of 4 independent experiments. Data represent the mean $\pm \mathrm{SD}$. ${ }^{*} P<0.05$, ${ }^{* *} P<0.001$, and ${ }^{* * *} P<0.0001$, by 2 -tailed $t$ test. Rel, relative. (C) H\&E-stained images of splenic sections on day 7 after immunization. Scale bars: $200 \mu \mathrm{m}$. (H) Representative immunofluorescence images show the expression of B220 (blue) and PNA (red) on splenic sections from WT and CD23-Rock2 mice and pooled quantifications of the average GC count per $\times 10$ field per mouse and the average GC size per mouse. Scale bars: $100 \mu \mathrm{m} . n=6$ per genotype. Data are from 3 independent experiments and represent the mean \pm SEM. ${ }^{* *} P<0.01$ and $P=0.10$, by Mann-Whitney $U$ test. DOWN, downregulated; UP, upregulated. 
A

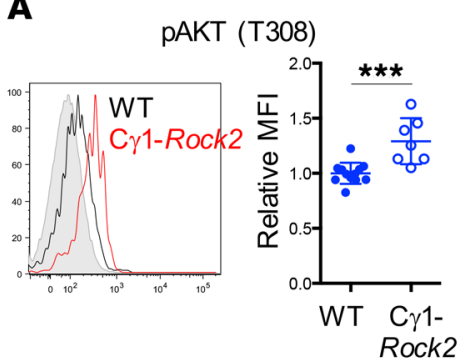

pAKT (S473)

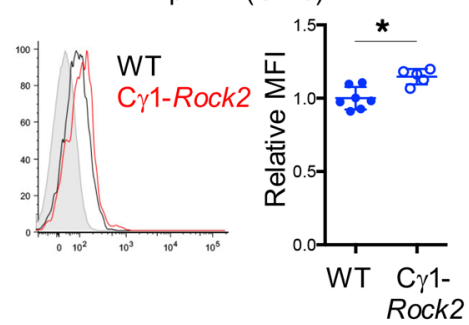

B

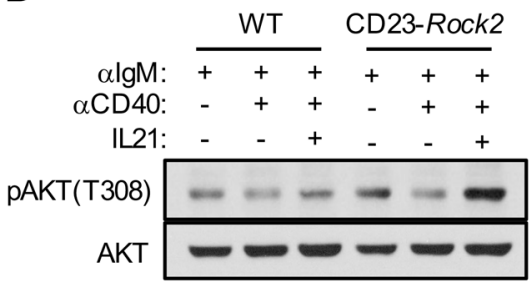

C

Enrichr upstream regulator analysis ROCK2-repressed gene set (ChEA 2016)

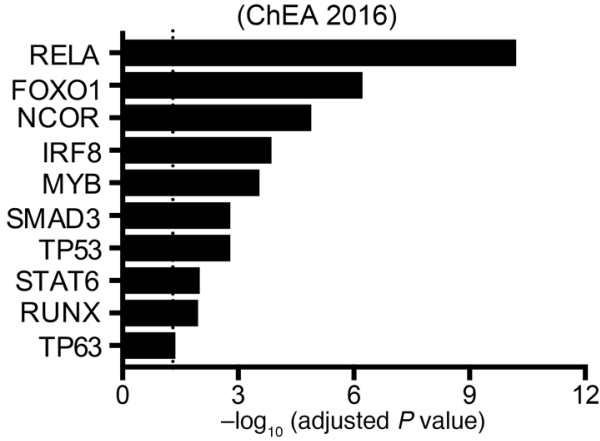

$\mathbf{F}$

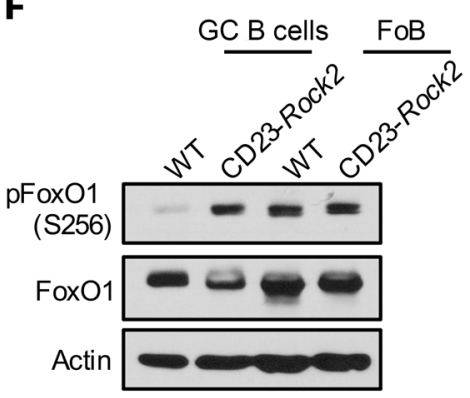

G

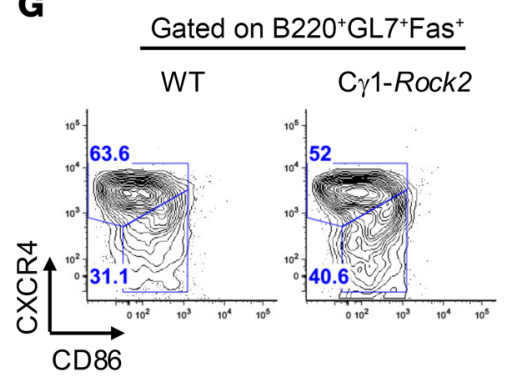

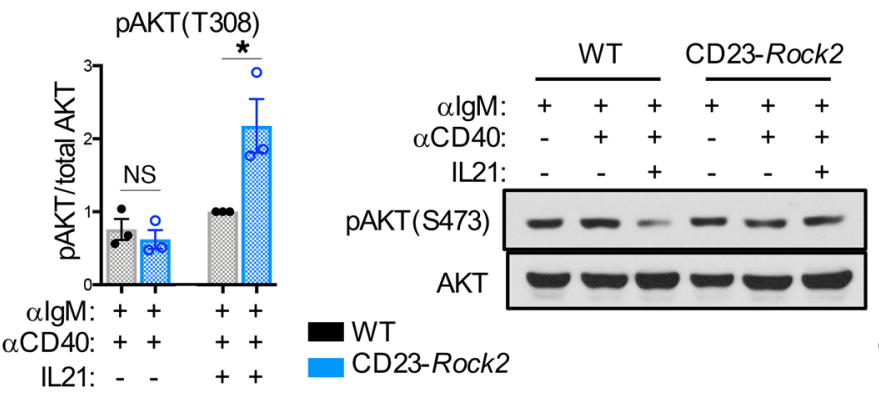

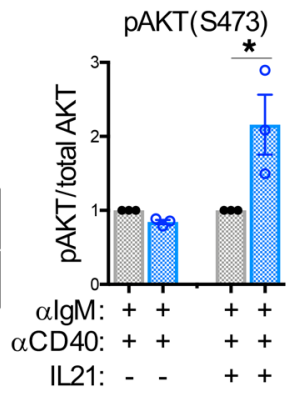

E FoxO1 GC B cell targets
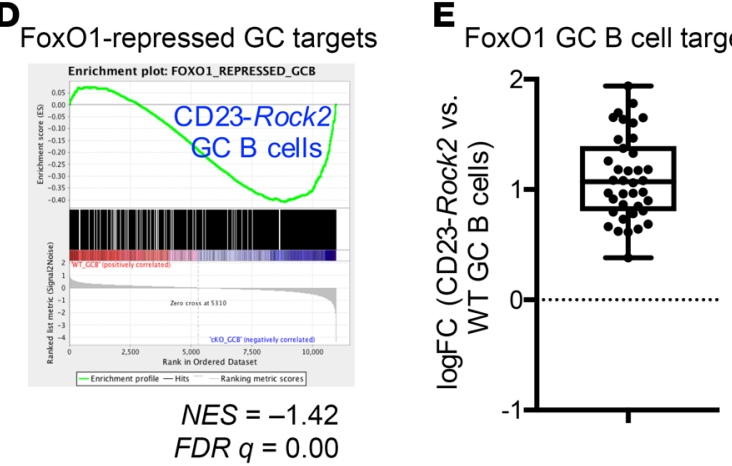

H

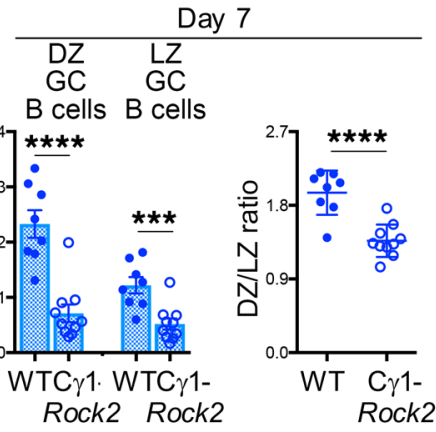

Figure 5. B cell ROCK2 regulates AKT activity and GC polarization. (A) Representative histograms and pooled quantifications of p-AKT (T308) and p-AKT (S473) in GC B cells from WT (black) and C $\gamma 1-R o c k 2$ (red) mice on day 7 after immunization. WT non-GC B cells (gray) are shown as a control. Quantifications were based on 3 independent experiments and were normalized to WT mice for each experiment. $n>6$. Data represent the mean \pm SEM. ${ }^{*} P<$ 0.05 and ${ }^{* *} P<0.001$, by unpaired, 2-tailed $t$ test. (B) Representative immunoblots and pooled quantifications of $p$-AKT (T308) and $p$-AKT (S473) from extracts of $\mathrm{CD} 23^{+} \mathrm{B}$ cells from WT and CD23-Rock2 mice cultured for 3 days as in Figure 1, A and B. Quantification was calculated as the densitometric ratio of $\mathrm{p}$-AKT to total AKT. $n=3$. Data are from 3 independent experiments and represent the mean $\pm \mathrm{SEM}$. ${ }^{*} P<0.05$, by unpaired, 2 -tailed $t$ test. (C) Upstream regulator analysis of genes upregulated in CD23-Rock2 GC B cells as determined by Enrichr analysis using the ChEA 2016 database. (D) GSEA plot shows enrichment of the FOX01-repressed gene set in CD23-Rock2 GC B cells. NES, normalized enrichment score. (E) Box plot shows the mean log FC of FOX01-repressed target genes significantly upregulated in CD23-Rock2 GC B cells. Each point represents the log FC of a different gene, with the whisker plot showing minimum to maximum values and the median. (F) Representative immunoblot of p-FOXO1 (S256) and total FOXO1 in sorted FoBs and GC B cells from the indicated mice on day 7 after immunization. Data are representative of 2 independent experiments. (G and $\mathbf{H})$ Representative FACS plots (G) and quantifications $(\mathbf{H})$ of DZ (CXCR4 ${ }^{\text {hi }} C D 86^{10}$ ) and LZ (CXCR4 $4^{\text {hi }}$ CD86 $\left.6^{10}\right)$ GC B cells from WT or $C \gamma 1-$ Rock2 mice on day 7 after immunization. $n>8$. Data are from 3 independent experiments and represent the mean \pm SEM. ${ }^{* *} P<0.001$ and ${ }^{* * * *} P<0.0001$, by unpaired 2 -tailed $t$ test. 


\section{A}

GL7 $7^{+} \mathrm{Fas}^{+} \mathrm{GC} B$ cells
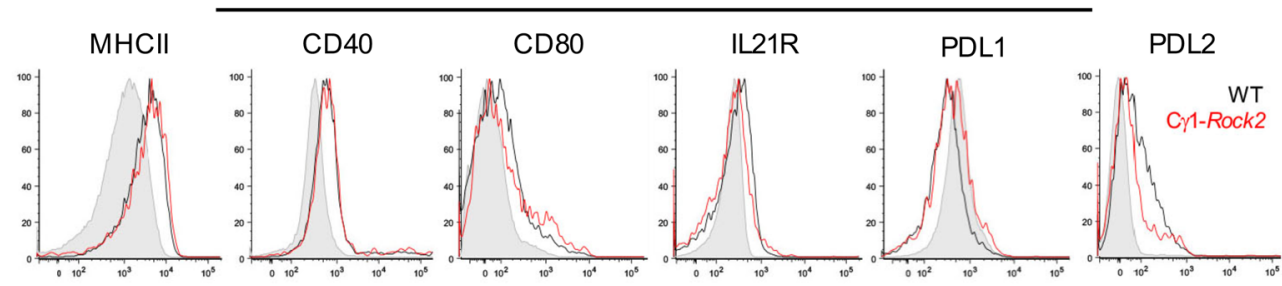

B
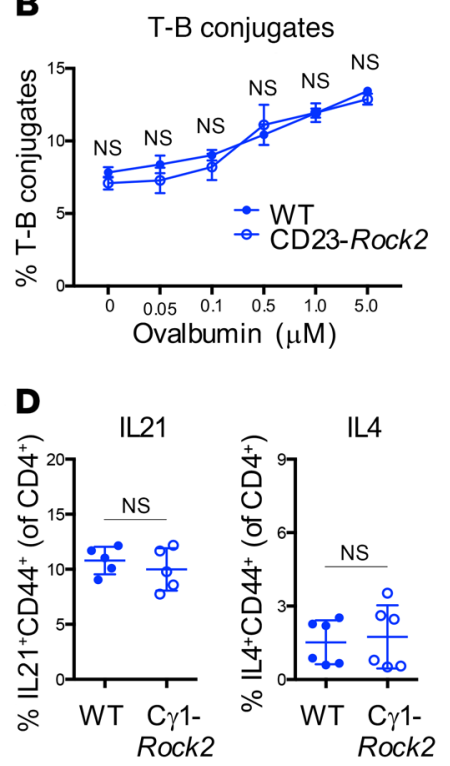

$\mathbf{F}$

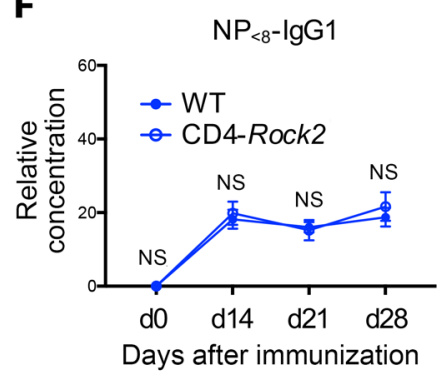

C

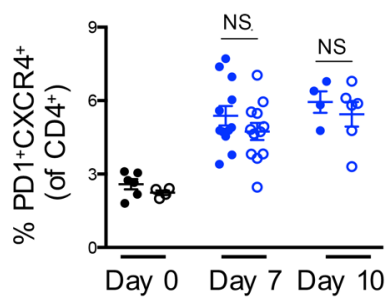

Follicular T helper cells
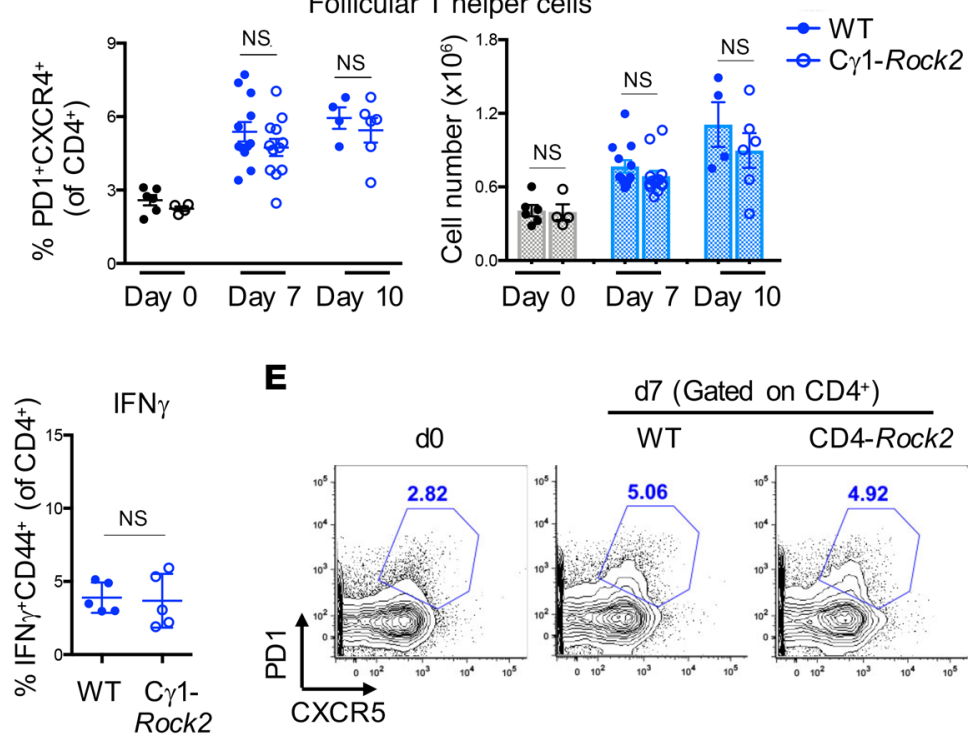

E

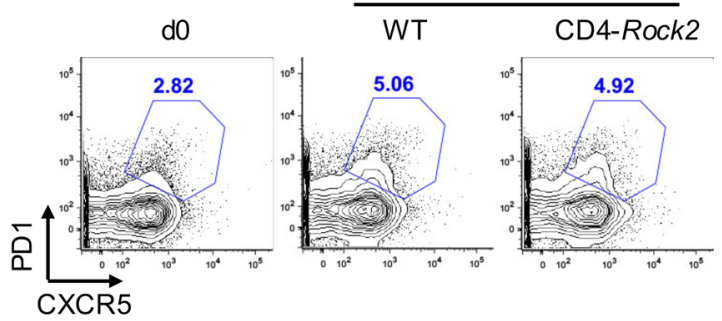

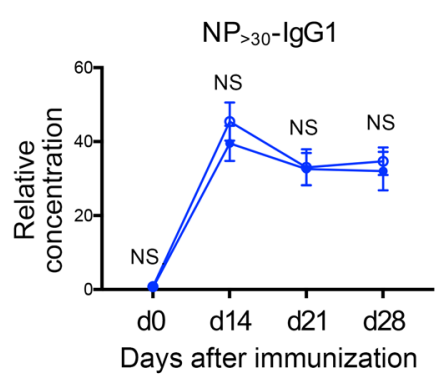

Figure 6. B cell ROCK2 is dispensable for T cell-B cell interactions and Tfh responses. (A) Representative histograms of the indicated markers on the surface of GC B cells from WT (black) or C $\gamma 1$-Rock2 (red) mice on day 7 after immunization. WT non-GC B cells (shaded gray) are shown as a control. Data are representative of at least 4 mice per genotype across 2 independent experiments. (B) Pooled quantification of B cell-T cell (T-B) conjugates from cultures of OT-II T cells with WT or CD23-Rock2 B cells loaded with OVA peptide (amino acids 323-339). $n=3$. Data are from 3 independent experiments and represent the mean \pm SEM. Statistical significance was determined by 2-way ANOVA followed by Holm-Šidák's test for multiple comparisons. (C) Quantifications of the frequency and number of Tfh cells (CD4+CXCR5 $\left.{ }^{+} P D 1^{+}\right)$after immunization. $n>4$. Data are from at least 2 independent experiments per time point and represent the mean \pm SEM. Statistical significance was determined by unpaired, 2 -tailed $t$ test. (D) Quantification of IL-21, IL-4, and IFN- $\gamma$ production by $\mathrm{CD}^{+} \mathrm{T}$ cells following stimulation of splenocytes from mice on day 10 after immunization with PMA and ionomycin. $n>5$. Data are from 2 independent experiments and represent the mean \pm SEM. Statistical significance was determined by unpaired, 2-tailed $t$ test. (E and $\mathbf{F})$ Rock $2^{f / f l}$ (WT) and CD4-Cre Rock2 $2^{f / f l}$ (CD4-Rock2) mice were immunized with $100 \mu \mathrm{g} \mathrm{NP-CGC} \mathrm{for} 7$ to 28 days. (E) Representative FACS plots of Tfh cells on day 7 after immunization. Data are representative of 4 mice per genotype across 2 independent experiments. (F) ELISA analysis of NP-specific IgG1 on days 0 to 28 after immunization. $n=4$. Data are representative of 2 independent experiments and indicate the mean \pm SEM. Statistical significance was determined by 2-way ANOVA followed by Holm-Šidák's test for multiple comparisons.

ure 5, $\mathrm{H}$ and I). Therefore, the absence of ROCK2 alone was insufficient to cause systemic dissemination of GC B cells, at least in young mice.

B cell ROCK 2 regulates AKT activity and GC polarization. Signaling downstream of several receptors important for GC responses, including GPCRs, results in AKT activation (41). Since the ROCKs have been shown to regulate the activity of various components of the AKT signaling pathway, including pyruvate dehydrogenase kinase 1 (PDK1) and phosphatase and tensin homolog (PTEN) (42, $43)$, we next investigated whether the absence of ROCK2 in GC 
A

Top GO pathwys down in CD23-Rock2 GC B cells
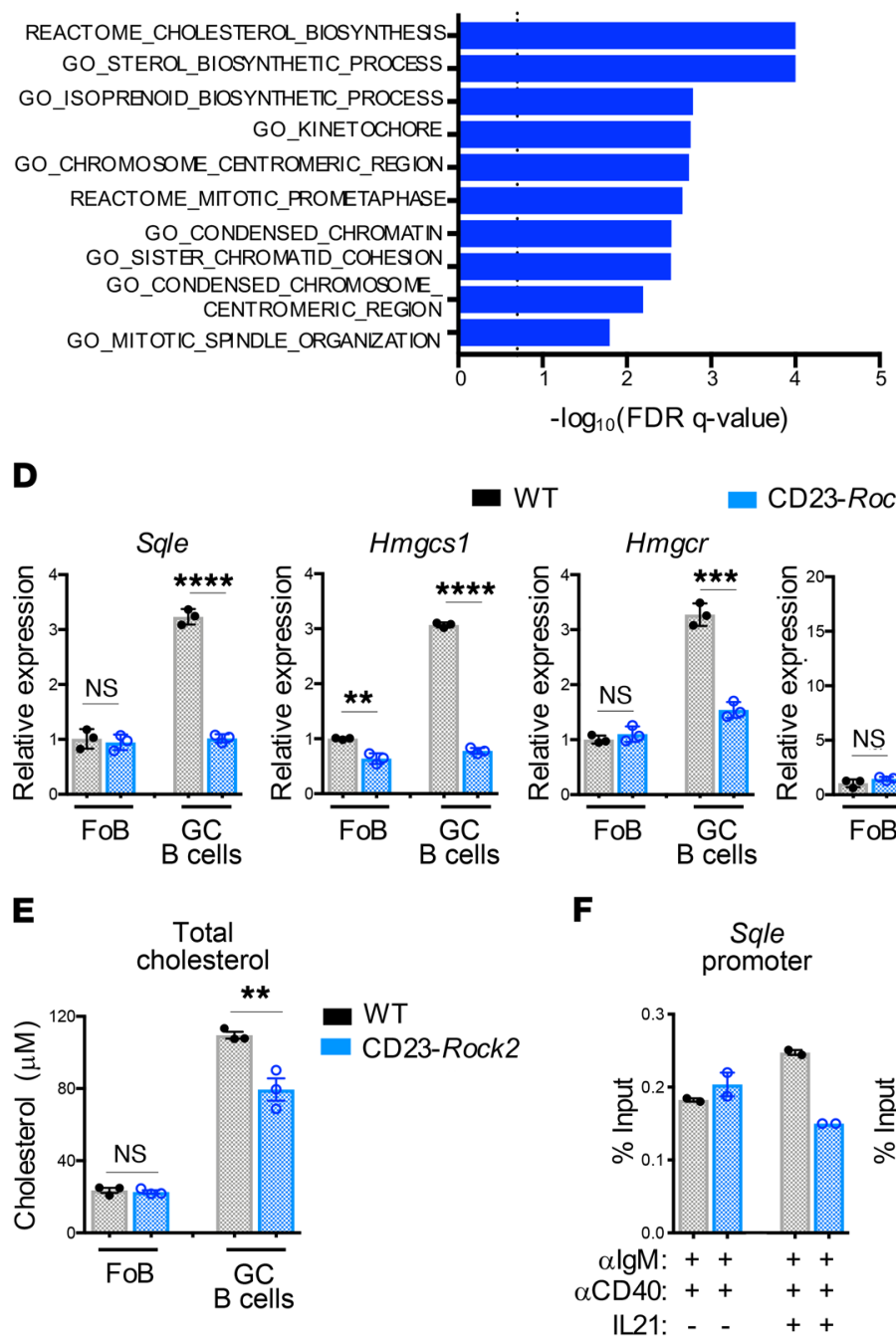

B

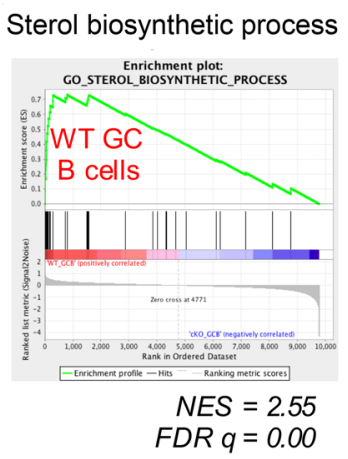

C

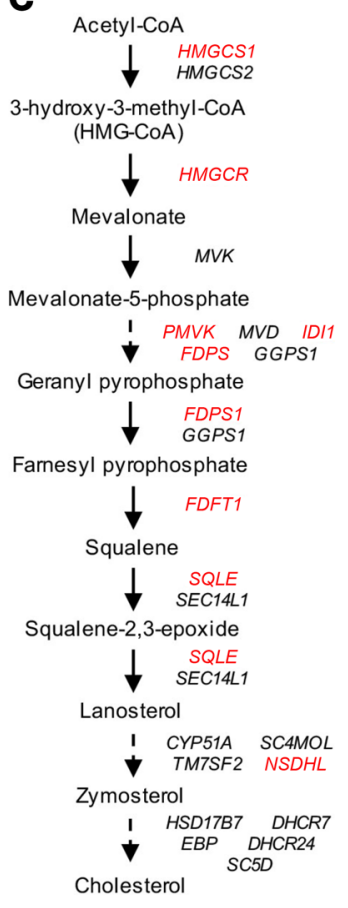

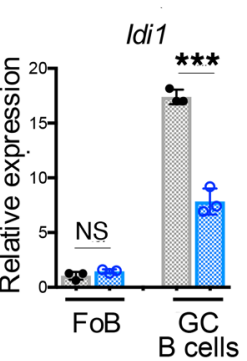
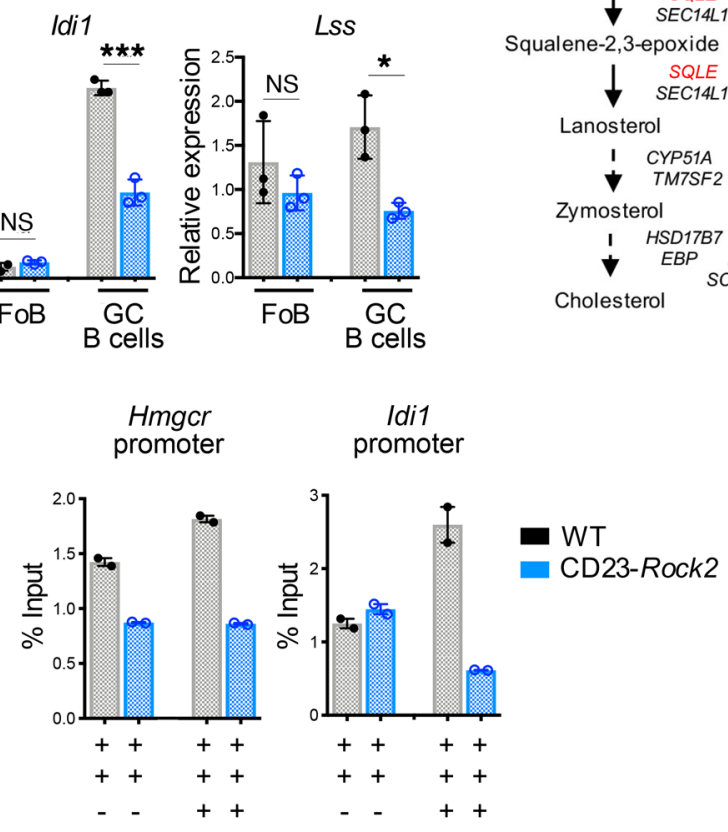

Figure 7. ROCK2 promotes cholesterol biosynthesis pathways in GC B cells by enhancing the activity of SREBP2. (A) Plot shows the top enriched gene sets downregulated in CD23-Rock2 GC B cells compared with WT GC B cells. Dotted line indicates significance cutoff at FDR $\underline{Q}=0.05$. (B) GSEA plot shows the enrichment of the GO_STEROL_BIOSYNTHETIC_PROCESS gene set in WT GC B cells. (C) Schematic shows the enzymes and intermediates involved in the cholesterol biosynthesis pathway. Enzymes highlighted in red are encoded by genes that contribute to the downregulation of the GO_STEROL_BIOSYNTHETIC_PROCESS gene set in CD23-Rock2 GC B cells (A). (D) Representative RT-qPCR analysis of the indicated genes in sorted FoBs or GC B cells from WT or CD23-Rock2 mice 7 days after immunization. $n=3$. Data are based on technical triplicates and are representative of 3 independent experiments. Data represent the mean $\pm \mathrm{SD}$. ${ }^{*} P<0.05$, ${ }^{* *} P<0.01$, ${ }^{* *} P<0.001$, and ${ }^{* * * *} P<0.0001$, by unpaired, 2 -tailed $t$ test. (E) Total cholesterol in sorted FoBs and GC B cells 7 days after immunization. $n=2$. Data are from 2 independent experiments and represent the mean $\pm S E M$. ${ }^{* *} P<0.01$, by unpaired, 2 -tailed $t$ test. (F) $\mathrm{CD}_{2} 3^{+} \mathrm{B}$ cells from WT (black) or CD23-Rock2 (blue) mice were purified and stimulated for 3 days as in Figure 1, A and B. Plots show representative data for ChIP-qPCR of SREBP2 binding to the indicated loci. Data are representative of 3 independent experiments and indicate the mean \pm SD.

B cells alters AKT activity. GC B cells from immunized C $\gamma 1-$ Rock2 mice had significantly higher p-AKT levels than did WT GC B cells (Figure 5A). In line with these findings, CD23-Rock2 B cells had higher levels of p-AKT than did WT B cells upon in vitro stimulation with anti-IgM, anti-CD40, and IL-21 (Figure 5B and Supplemental Figure 6A). These findings show that ROCK2 restrained AKT activation in GC B cells.

One of the key targets of AKT is FOXO1 (41), a transcription factor that is critically important for the partitioning of GC B cells between the dark and light zones (11-13). AKT-dependent phosphorylation of FOXO1 results in its cytoplasmic sequestration and inhibition of its transcriptional activity (41). The increased AKT activity in B cells lacking ROCK2 suggested that the ROCK2-controlled transcriptional program might include FOXO1 target genes. An upstream regulator analysis using the ChIP Enrichment Analysis (ChEA) 2016 database from Enrichr indeed identified FOXO1 as one of the top potential regulators of the ROCK2repressed gene set in GC B cells (Figure $5 C$ ). GSEA, furthermore, revealed that FOXO1-repressed GC targets (11) were upregulated in CD23-Rock2 GC B cells compared with WT GC B cells (Figure 5, D and E, and Supplemental Figure 6B), suggesting that these genes are normally also inhibited by ROCK2. In line with these 
A

Peaks at ROCK2-regulated genes

\begin{tabular}{ccc} 
Motif & Best match & $P$ value \\
\hline ACGAAgI PU.1 (ETS) & $1 \times 10^{-138}$
\end{tabular}

CCAGAGEGGE CTCFL $1 \times 10^{-135}$

$=\triangleq T$ TTCAT POU2F2 $1 \times 10^{-70}$

\begin{tabular}{|c|c|c|}
\hline AAcIGAAA & IRF8 & $1 \times 10^{-39}$ \\
\hline$=-\operatorname{sen} s$ & KLF5 & $1 \times 10^{-18}$ \\
\hline
\end{tabular}

C

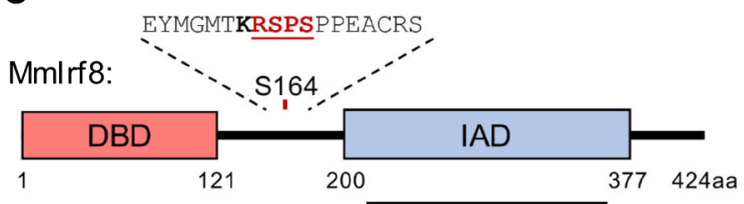

HsIRF8:

PU.1

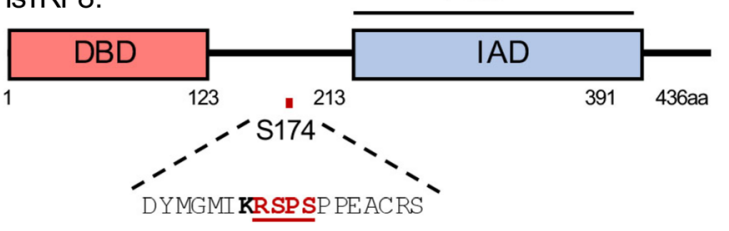

B
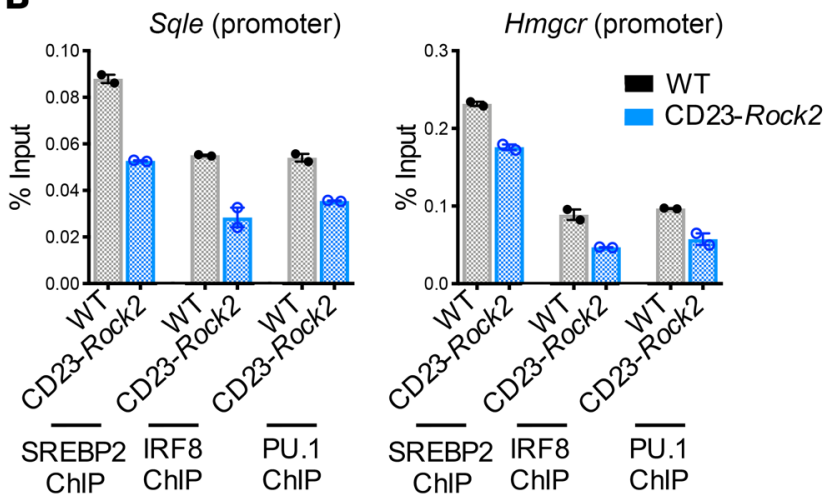

D

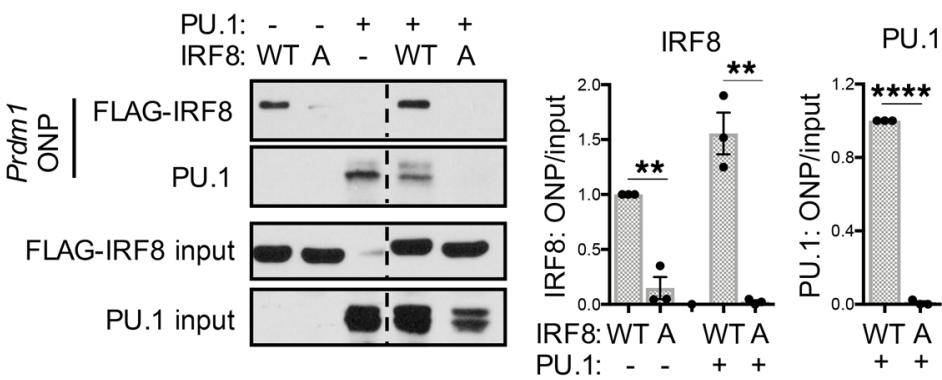

E
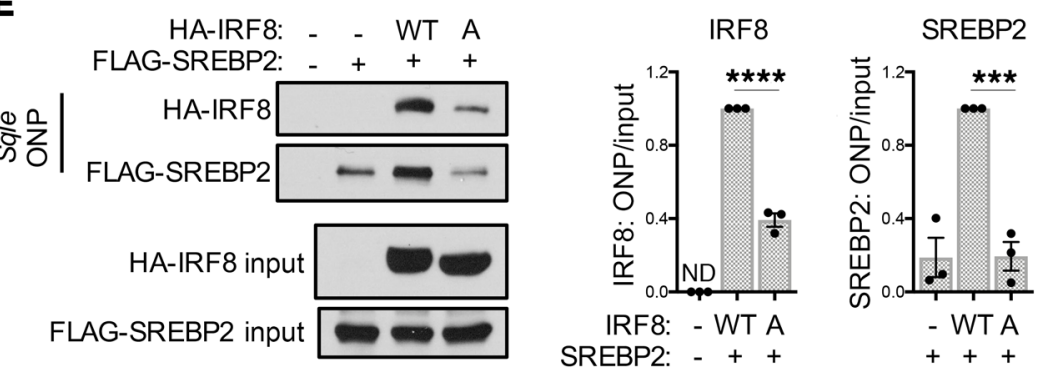

$\mathbf{F}$
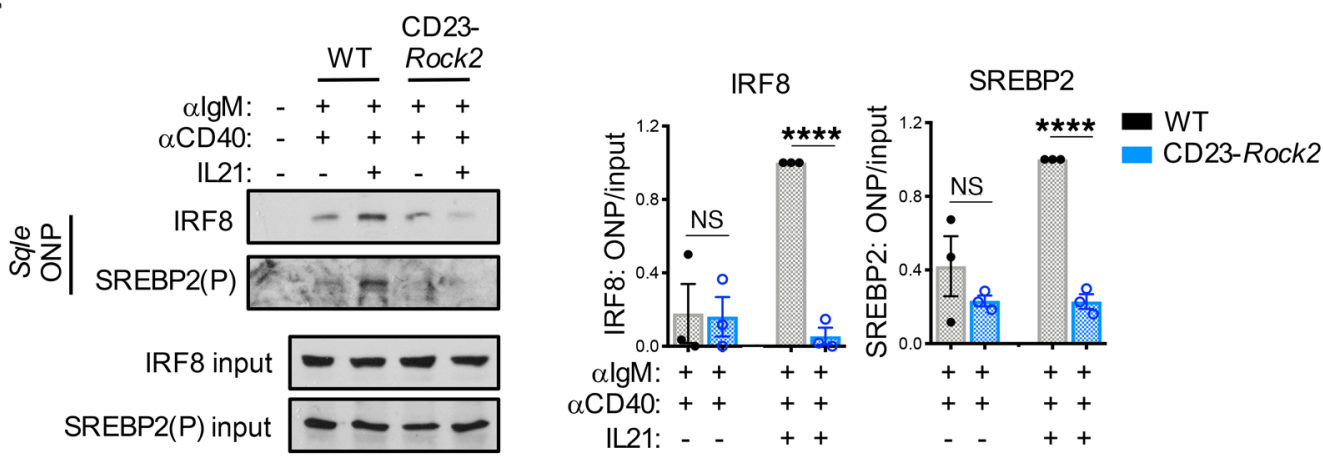

Figure 8. ROCK2 phosphorylates IRF8 and promotes its cooperation with SREBP2. (A) Plot shows the top-overrepresented motifs from a de novo motif analysis of peaks at loci of differentially expressed genes between WT and CD23-Rock2 GC B cells. (B) Representative results for ChIP-qPCR of SREBP2, IRF8, and PU.1 binding to the indicated loci. Data are representative of 3 independent experiments and indicate the mean \pm SD. (C) Schematic shows the location of the ROCK phosphorylation consensus sequence on the mouse (MmIrf8) and human (HsIRF8) IRF8 protein. (D and E) 293T cells were transfected with constructs expressing PU.1, FLAG-tagged SREBP2, and either FLAG- or HA-tagged WT IRF8 (WT) or a mutant of IRF8 (A) at S164. ONP assays of nuclear extracts from 293T cells assessed with biotinylated oligonucleotides from a Prdm1 intronic region (D) or a Sqle promoter region (E) followed by immunoblot analysis of precipitated proteins or inputs. Quantification shows the ratio of precipitated protein to input protein. $n=3$. Data represent the mean \pm SEM. ${ }^{* *} P<0.01$, ${ }^{* * *} P$ $<0.001$, and ${ }^{* * *} P<0.0001$, by unpaired, 2-tailed $t$ test $(\mathbf{D})$ or 1-way ANOVA followed by Tukey's test for multiple comparisons (E). Lanes in $\mathbf{D}$ were run on the same gel but were noncontiguous. ND, not detected. (F) ONP assay of nuclear extracts from WT or CD23-Rock2 B cells following stimulation for 3 days as in Figure 1, A and B, using biotinylated oligonucleotides from a Sqle promoter. Quantification shows the ratio of precipitated protein to input protein. $n=3$. Data represent the mean \pm SEM. ${ }^{* * * *} P<0.0001$, by 1-way ANOVA followed by Tukey's test for multiple comparisons. 
findings, we observed a marked increase in FOXO1 phosphorylation in sorted GC B cells from CD23-Rock2 mice compared with WT GC B cells (Figure 5F). In addition, we detected lower levels of FOXO1 protein known to accompany its phosphorylation upon gating either total GC B cells or LZ B cells (Supplemental Figure 6, $\mathrm{C}$ and $\mathrm{D}$, and ref. 44). Thus, ROCK2 promotes FOXO1 activation and controls a FOXO1-repressed gene set in GC B cells, probably through its ability to regulate AKT activity.

Mice with B cell-specific manipulations of components of the AKT pathway, including AKT, PTEN, and FOXO1, exhibit alterations in GC polarity including loss of the DZ (11-13). To assess whether ROCK2 could also contribute to GC partitioning, we first evaluated the frequencies of GC B cell subpopulations by examining the expression of CXCR4 and CD86 on the surface of GC B cells (Figure 5G and Supplemental Figure 6E). Consistent with decreases in the number of total GC B cells, immunized C $\gamma 1$ Rock2 and CD23-Rock2 mice had reduced numbers of both DZ $\left(\mathrm{CXCR} 4^{\mathrm{hi}} \mathrm{CD} 86^{\mathrm{lo}}\right)$ and $\mathrm{LZ}\left(\mathrm{CXCR} 4^{\mathrm{lo}} \mathrm{CD} 86^{\mathrm{hi}}\right) \mathrm{GC}$ B cells compared with WT mice (Figure 5H and Supplemental Figure 6F). The effects on DZ GC B cells were, however, more pronounced than those on LZ GC B cells, and GCs from either C $\gamma 1$-Rock2 or CD23-Rock2 mice had an increased frequency of LZ GC B cells and a decreased DZ/LZ GC B cell ratio (Figure 5H and Supplemental Figure 6F). Immunofluorescence staining also showed abnormalities in the DZ/LZ ratio, in which CD23-Rock2 mice contained a smaller DZ $\left(\mathrm{CD}^{-} 5^{-} \mathrm{IgD}^{-}\right)$than did WT mice, with the GC LZ $\left(\mathrm{CD}^{-} 5^{+} \operatorname{IgD}^{-}\right)$ comprising the majority of the GC structure (Supplemental Figure 6, G and $\mathrm{H}$ ). These findings show that the absence of ROCK2 in B cells resulted in skewed DZ/LZ GC B cell ratios.

$B$ cell ROCK 2 is dispensable for $T$ cell-B cell interactions and $T f h$ responses. Since reciprocal signals between $B$ cells and $\mathrm{T}$ cells are required for productive humoral responses $(1,3)$, we next assessed the role of $\mathrm{B}$ cell ROCK 2 in mediating $\mathrm{T}$ cell-B cell interactions. Although surface expression levels of MHC class II (MHCII), CD40, CD80, and IL-21R were mostly similar between C $\gamma 1-$ Rock2 and WT GC B cells, we observed some alterations in the expression of PD1 ligands in C $\gamma 1-$ Rock2 GC B cells compared with expression levels in WT GC B cells (Figure 6A and Supplemental Figure 7A). To directly examine whether B cell ROCK2 is involved in mediating the interactions of $\mathrm{B}$ cells with $\mathrm{T}$ cells, we used conjugate assays to monitor the interaction of OVA-pulsed WT and CD23-Rock2 B cells with activated OT-II T cells expressing an OVA-specific T cell antigen receptor, as previously described (6). We observed no differences in the ability of WT and ROCK2deficient $\mathrm{B}$ cells to form conjugates with T cells (Figure 6B). These data suggest that the presence of ROCK 2 in $\mathrm{B}$ cells is not required to mediate in vitro conjugate interactions of $\mathrm{B}$ cells with $\mathrm{T}$ cells.

In line with the ability of ROCK2-deficient B cells to form conjugates normally with $\mathrm{T}$ cells, the frequencies and numbers of Tfh cells in immunized WT and C $\gamma 1-$ Rock2 and CD23-Rock2 mice were similar, and these cells displayed comparable levels of BCL6, ICOS, and CXCR5 (Figure 6C and Supplemental Figure 7, B-F). No differences in the production of IL-21, IL-4, or IFN- $\gamma$ (Figure 6D and Supplemental Figure 7, G-I) or in the localization of $\mathrm{T}$ cells within the GCs (Supplemental Figure 7J) were detected in mice lacking ROCK2 in B cells. Since the ratio of $\mathrm{T}$ follicular regulatory ( $\mathrm{Tfr}$ ) to $\mathrm{Tfh}$ cells can correlate with the magnitude of antibody responses (45), we also assessed the frequency of Tfr cells, which was unchanged in C $\gamma 1-$ Rock2 mice (Supplemental Figure 7K). We found that B cell-specific ROCK2 was dispensable for the proper generation and function of follicular T cell subsets. Given the importance of B cell ROCK2 in mediating optimal GC and humoral responses, we next assessed the requirement for $\mathrm{T}$ cell ROCK 2 in mediating TD responses by crossing Rock $2^{f / f l}$ mice with CD4-Cre-expressing mice. CD4-Cre Rock $2^{f l / f l}$ (termed CD4-Rock2) or control Rock2 $2^{f / f l}$ (WT) mice were immunized with NP-CGG. We observed no differences in Tfh cell frequencies or numbers in CD4-Rock2 mice compared with WT mice (Figure 6E). Total and antigen-specific GC B cell responses were also unaffected in CD4-Rock2 mice (Supplemental Figure 8, A-D). Furthermore, CD4-Rock2 mice exhibited similar levels of NP-specific antibodies (Figure 6F). Therefore, in contrast to the findings with B cell ROCK2, expression of ROCK2 in T cells was not required for efficient TD humoral responses.

ROCK2 induces expression of the cholesterol biosynthesis pathway in GC B cells by enhancing the activity of SREBP2. In addition to mediating inhibitory effects in GC B cells, genome-wide transcriptional analysis revealed that a smaller set of targets was downregulated in CD23-Rock2 GC B cells (Figure 4C). Remarkably, among the top downregulated pathways were those related to sterol and cholesterol biosynthesis (Figure 7, A-C, and Supplemental Figure 9A). Downregulation of several key enzymes involved in cholesterol biosynthesis, including squalene epoxidase (Sqle), 3-hydroxy-3-methylglutaryl-CoA synthase 1 (Hmgcs1), 3-hydroxy-3-methylglutaryl-CoA reductase ( $\mathrm{Hmgcr}$ ), isopentenyldiphosphate delta isomerase 1 (Idi1), and lanosterol synthase (Lss), was confirmed by RT-qPCR in GC B cells from CD23-Rock2 mice (Figure 7D). Consistent with these findings, total cholesterol levels were significantly reduced in GC B cells from CD23-Rock2 mice compared with those from WT GC B cells (Figure 7E). We found that the expression of these targets was also decreased in CD23-Rock2 B cells following in vitro stimulation with anti-IgM, anti-CD40, and IL-21 (Supplemental Figure 9B). Expression of the low-density lipoprotein receptor $(\mathrm{Ldll})$ was also lower in CD23Rock2 GC B cells than in GC B cells from WT mice (Supplemental Figure 9C). Taken together, these data suggest that ROCK2 promotes cholesterol biosynthesis in GC B cells.

SREBP2 is the major transcriptional regulator of sterol synthesis and directly induces the expression of enzymes involved in the mevalonate pathway (46). In response to cholesterol starvation, SREBP2 is activated by a complex process involving its transport from the endoplasmic reticulum to the Golgi, where it is cleaved, followed by the translocation of transcriptionally active fragments to the nucleus (46). Transcript expression of Srebf1 and Srebf2, the genes encoding SREBP1 and SREBP2, respectively, was similar in CD23-Rock2 GC B cells compared with expression in WT GC B cells (Supplemental Figure 9D). Furthermore, we did not detect any significant differences in the proteolytic processing of SREBP2 in CD23-Rock2 B cells compared with WT B cells (Supplemental Figure 9E). To directly assess whether ROCK2 could promote the transcriptional activity of SREBP2, we next performed ChIP assays with an anti-SREBP2 antibody followed by RT-qPCR using primers encompassing regulatory sites previously shown to be bound by SREBP2 (47). These experiments demonstrated that 
the binding of SREBP2 to the promoters of Sqle, Hmgcr, and Idi1 was decreased in CD23-Rock2 B cells compared with that seen in WT B cells (Figure 7F). Taken together, these data suggest that, in GC B cells, ROCK2 regulates the cholesterol biosynthesis pathway by enhancing SREBP2 transcriptional activity.

ROCK 2 phosphorylates IRF 8 and promotes its cooperation with $S R E B P 2$. To gain further insights into the mechanisms by which ROCK 2 controls the expression of key enzymes in the mevalonate pathway in GC B cells, we next performed ATAC-Seq on GC B cells from CD23-Rock2 mice. We selected 2300 peaks that were found in $-10 \mathrm{~kb}:+200 \mathrm{bp}$ regions around the transcription start sites of differentially expressed genes $(P<0.01$, fold change [FC] $>2$ ) between CD23-Rock2 and WT GC B cells. We then determined the transcription factor-binding motifs overrepresented in these peaks (Figure 8A). The top motif found in ATAC-Seq peaks from the differentially expressed genes corresponded to members of the ETS family of transcription factors, including PU.1 (Figure $8 \mathrm{~A}$ ), which promotes the transcriptional profile of GC $\mathrm{B}$ cells through interactions with IRF8 $(7,10)$. IRF8-binding sites were also significantly overrepresented in the motif analysis (Figure 8A). To evaluate whether promoters bound by SREBP2 could also be targeted by IRF8 and PU.1, we performed ChIP-qPCR assays with antibodies against IRF8 or PU.1 (Figure 8B). In addition to SREBP2, we also observed binding of both IRF8 and PU.1 to the promoters of Sqle and Hmgcr (Figure 8B). Targeting of IRF8 and PU.1 to these sites was decreased in CD23-Rock2 B cells (Figure $8 \mathrm{~B}$ ). These findings show that ROCK2 regulated the ability of IRF8 and PU.1 to bind to the promoters of Sqle and Hmgcr.

Since we previously demonstrated that ROCK2 can phosphorylate and regulate the activity of IRF4 during Th17 differentiation (28), we next investigated the possibility that ROCK2 could also phosphorylate and regulate the activity of IRF8. Given that IRF8 is a $50-\mathrm{kD}$ a protein, we performed in vitro kinase assays using a deletion mutant of IRF8 that lacks the amino-terminal DNA-binding domain (IRF8 $\Delta 1-150$ ) to avoid overlap of IRF8 protein with the heavy chain of the precipitating antibody. Extracts from 293T cells transfected with the IRF8 $\Delta 1-150$ mutant were immunoprecipitated with an IRF8 antibody and incubated with purified, constitutively active ROCK2. IRF8 phosphorylation upon incubation with the constitutively active ROCK2 protein was detected using a phospho-serine antibody (Supplemental Figure 10A). An examination of the murine IRF8 protein revealed the presence of a potential ROCK2 consensus site at S164, which is located in the linker domain of IRF8 and is conserved in murine and human IRF8 (Figure 8C and Supplemental Figure 10B). To assess whether the phosphorylation of IRF8 regulates its activity, we generated an IRF8 mutant in which S164 was mutated to alanine (IRF8A). We next examined the ability of IRF8A to bind DNA by oligonucleotide precipitation (ONP) assay using an oligonucleotide representing the Prdm1 intronic region, a classic target of IRF8 and PU.1 in B cells (7). WT IRF8 strongly bound the Prdm1 oligonucleotide either alone or in combination with PU.1 (Figure 8D). In contrast, the IRF8A mutant exhibited a significantly reduced ability to bind to the Prdm1 oligonucleotide (Figure 8D). Cotransfection of the IRF8A mutant together with PU.1 also interfered with the ability of PU.1 to bind to this site (Figure 8D). Thus, IRF8 phosphorylation facilitated its DNA binding and its interaction with PU.1.
We next performed ONP assays using an oligonucleotide from the Sqle promoter to assess the impact of IRF8 phosphorylation on binding to this target site. In contrast to WT IRF8, binding of IRF8A to the Sqle oligonucleotide was significantly decreased (Figure 8E). Expression of IRF8A also decreased the ability of SREBP2 to bind to this site, suggesting that the phosphorylation of IRF8 is required for optimal targeting of SREBP2 to the Sqle promoter (Figure 8E). Furthermore, when we subjected extracts from either WT or CD23-Rock2 B cells stimulated in vitro to ONP assays with the Sqle oligonucleotide, we found that endogenous IRF8 could bind to this site and that binding of both IRF8 and SREBP2 was decreased in the absence of ROCK2 (Figure 8F). Taken together, these data suggest that ROCK2, by regulating IRF8 phosphorylation, promotes the targeting of SREBP2 and IRF8 transcriptional complexes to the promoters of key enzymes in cholesterol biosynthesis.

\section{Discussion}

Efficient GC responses require a complex interplay of signals that regulate $\mathrm{B}$ cell positioning, differentiation, and metabolic reprogramming (1-3). The molecular mechanisms used by B cells to coordinate these dynamic responses remain incompletely understood. Here, we demonstrate that the Rho kinase family member ROCK2 was activated in B cells in response to key $\mathrm{T}$ cell-derived signals and was required for efficient GC responses. Although ROCK2 activity in B cells was induced upon activation, high levels of ROCK1 activity in B cells could be observed irrespective of the stimulatory conditions, suggesting that the activation of ROCK1 and ROCK2 in the $\mathrm{B}$ cell compartment was differentially regulated. The existence of different multiprotein complexes where ROCK1 and ROCK2 are coupled with distinct guanine nucleotide exchange factors (GEFs) may potentially underlie these differences, since several different GEFs can activate RHOA (48). We show that ROCK2 regulated GC formation and maintenance by controlling a unique transcriptional program that promoted the proper positioning and metabolic fitness of GC B cells. These effects were associated with the ability of ROCK2 to inhibit AKT activation and promote a FOXO1-dependent transcriptional program. Notably, we also found that ROCK2 regulated the expression of key cholesterol synthesis enzymes in GC $\mathrm{B}$ cells by enhancing the targeting of SREBP2 to their promoters in a manner that depended on the ROCK2-mediated phosphorylation of IRF8. These studies thus suggest that ROCK2 can function as a multifaceted regulator of GC B cell responses.

Proper positioning of $\mathrm{B}$ cells within a follicle is an essential requirement for the formation and function of GCs (2). B cell trafficking and localization within the follicle is regulated by a complex set of guidance cues that includes gradients of chemokines and lipid mediators that are sensed by multiple GPCRs (2, 3). Expression of Gpr183/EBI2 and CCR7 mediates the attraction of B cells toward the outer region of the follicle, and downregulation of their expression after cognate interactions with $\mathrm{T}$ cells is important for B cell positioning at the center of the follicle $(15,17$, 49). The ability of ROCK2 to regulate the expression of these and other GPCRs thus suggests that ROCK2 supports a transcriptional program that promotes the confinement of early GC B cells within the follicle. Interestingly, other key receptors that promote GC confinement, like S1PR2 and P2RY8, act via a Ga13/RHOA axis (2) and would thus likely activate the ROCKs by their actions on cyto- 
skeletal dynamics. Hence, the ROCK2-controlled transcriptional program may complement the ROCK-mediated cytoskeletal functions, enabling precise regulation of GC B cell positioning.

In addition to helping regulate $B$ cell localization within the follicle, we found that ROCK2 also contributed to the proper partitioning of these cells between the GC DZ and LZ, and its absence resulted in defective GC DZ formation. Interestingly, B cells lacking ROCK2 exhibited enhanced AKT activation, and the transcriptional program repressed by ROCK2 in GC B cells overlapped with the FOXO1-regulated gene set. Although we cannot exclude the possibility that the skewed DZ/LZ ratios observed in the absence of ROCK2 may have contributed to these findings, the marked increases in FOXO1 phosphorylation detected in ROCK2deficient GC B cells suggest that these effects were due to the ability of ROCK2 to inhibit AKT activation and thereby control FOXO1 activity. Recent reports have shown that the BCR- and CD40-driven AKT signaling networks in naive and GC B cells are differentially regulated in order to ensure optimal B cell selection in GCs $(13,22)$. Interestingly, enhanced AKT activation in ROCK2-deficient B cells was particularly noticeable upon stimulation with IL-21, suggesting that ROCK2 could limit the activation of AKT and likely promote the nuclear translocation of FOXO1 upon the productive interaction of a GC B cell with a Tfh cell. Upregulation of FOXO1 activity, in turn, would be expected to facilitate the acquisition of a DZ GC phenotype, leading to reentry of positively selected GC B cells into the DZ for further rounds of affinity maturation. Activation of ROCK 2 in GC B cells may therefore constitute another important layer in the complex signaling rewiring that GC B cells undergo during the selection process.

The establishment and maintenance of GC responses depend on productive collaborations between $\mathrm{T}$ and $\mathrm{B}$ cells, whereby $\mathrm{T}$ cell-derived signals promote the GC B cell phenotype and B cellderived signals reinforce the Tfh phenotype $(1,3)$. Surprisingly, $\mathrm{Tfh}$ cell differentiation and cytokine production were unaffected in mice with B cell-specific ROCK2 deletion. The lack of ROCK2 in $\mathrm{B}$ cells, furthermore, did not impair T cell-B cell conjugate formation, suggesting minimal requirements for B cell ROCK2 during the physical interaction of a B cell with a $\mathrm{T}$ cell in vitro, although we cannot exclude the possibility that ROCK2-deficient B cells failed to form effective cognate interactions with $\mathrm{T}$ cells in vivo. Surprisingly, mice lacking ROCK 2 in T cells also exhibited normal frequencies and numbers of Tfh and GC B cells upon immunization. Given that administration of a selective ROCK2 inhibitor in autoimmune mouse models decreased the numbers of Tfh cells $(30,50)$, simultaneous targeting of ROCK2 in multiple compartments may be required to uncover its role in Th responses. Alternatively, and not mutually exclusively, the pathogenic features of Tfh cells in autoimmune settings may render them more sensitive to manipulations of ROCK2 activity levels.

Maintenance of established GCs require B cells to adapt and survive in microenvironments with limited nutrient availability (18). Strikingly, our studies have uncovered an important role for ROCK2 in controlling the expression of key cholesterol biosynthesis enzymes in GC B cells. The ability of GC B cells to control de novo cholesterol biosynthesis via ROCK2 is likely to provide them with several advantages due to both the structural and signaling roles of these lipids. Indeed, given that cholesterol is a crit- ical component of plasma membranes, controlling its production could help meet the increased demands for membrane biosynthesis that GC B cells face during their rapid growth and terminal differentiation. Cholesterol homeostasis in B cells is also probably essential for lipid raft maintenance and the proper propagation of signals that drive B cell selection, such as those mediated by the BCR (51). Interestingly, AKT signaling has been shown to promote cholesterol biosynthesis (52), suggesting that ROCK2 modulates AKT signaling and cholesterol biosynthesis via independent mechanisms. Of note, one of the critical downstream steps in the mevalonate pathway is the generation of intermediates like geranylgeranyl pyrophosphate that are necessary for the prenylation and activation of small GTPases such as RHOA (48). Thus, by controlling de novo cholesterol biosynthesis, ROCK2 could also promote activation of the RHOA family of small GTPases and thus reinforce its own activation, generating a powerful positive feedback loop. Given the complex biology of this family, detailed biochemical approaches beyond ELISAs will be required to dissect the specific role of ROCK 2 in this process.

Our studies showed that ROCK2 modulated the expression of the mevalonate pathway in B cells via a mechanism whereby the activity of SREBP2 was enhanced by its interaction with a critical controller of GC B cells, IRF8, thereby enabling cholesterol biosynthesis to be regulated in a lineage-selective manner. These findings suggest that, in addition to promoting the expression of classical GC target genes such as Bcl6 and Aicda (6, 7, 9, 10), IRF8 also plays important roles in modulating the metabolic reprogramming of GC B cells. SREBP2-IRF8 interaction is dependent on the phosphorylation of a serine residue on IRF8, which is located in the linker region of IRF8 and would be expected to affect both its DNA binding activity and cofactor interactions, which is indeed what we observed. Phosphorylation of IRF8 also affected its interaction with PU.1, which likely contributes to the finding that TD responses were more profoundly decreased in ROCK2-deficient mice as compared with mice lacking IRF8 alone $(7,8,10)$. Although ROCK2 can also phosphorylate IRF4 (28), the known downregulation of IRF4 expression observed in GC B cells (4) renders IRF8 the relevant physiological target in GC B cells. Thus, we predict that the functional outcome of the ROCK2-mediated actions in $\mathrm{B}$ cells will be dictated not only by the presence of signals like CD40 and IL-21, which control ROCK2 activity, but also by the differential availability of IRF family members. Interestingly, high levels of IRF8 are also observed in myeloid cells and reactive microglia and, like the ROCKs, IRF8 has been linked to vascular inflammation and cardiovascular disease $(25,53,54)$, raising the possibility that the role of IRF8 as a coregulator of SREBP2 function may not be restricted to $\mathrm{B}$ cells and that ROCK-IRF8-SREBP crosstalk may play a broader role in inflammatory responses.

Our studies identify ROCK2 as a multifaceted regulator of GC B cell responses, which can help B cells to rapidly integrate their positioning and metabolic requirements during the complex organizational changes that underlie GC formation and maintenance. Given the availability of a wide range of ROCK inhibitors $(24,25)$, including selective ROCK 2 inhibitors and statins, as well as recent studies highlighting the use of the mevalonate pathway as a vaccine adjuvant (55), the involvement of ROCK2 in the regulation of GC responses could have immediate and broad therapeutic implica- 
tions. Since alterations in upstream regulators of the RHOA pathway like ARHGEF1 have emerged as key abnormalities in lymphomagenesis, a detailed understanding of whether these alterations are linked to deficiencies in ROCK2 activation will be critical to safely harness the therapeutic potential of this pathway.

\section{Methods}

Mice. The generation of Rock $2^{f / f l}$ and CD23-Cre mice was previously described $(34,56)$. C57BL/6, B6.Cg-Tg(Cd4-Cre)1Cwi/BfluJ (CD4$\mathrm{Cre})$, and B6.129P2(Cg)-Ighg1 ${ }^{\operatorname{ml} 1(\mathrm{Cre}) \mathrm{Cgn}} / \mathrm{J}(\mathrm{C} \gamma 1-\mathrm{Cre})$ mice were obtained from The Jackson Laboratory. All mice were on a C57BL/6 background. Male and female mice between 6 and 10 weeks of age were used in all experiments unless otherwise noted. All the mice used in the experiments were housed in a specific pathogen-free animal facility at HSS and Weill Cornell Medical College.

Immunizations, cell sorting, and flow cytometry. Mice were immunized with $100 \mu \mathrm{g} \mathrm{NP}_{30-40}$-CGG in alum 0 to 28 days before analysis. For cell sorting, single-cell suspensions from pooled spleens were preenriched for B cells with biotinylated anti-B220 and streptavidin microbeads. B cells stained with B220, CD23, CD38, and GL7 were sorted on either a BD FACS Aria II or a BD Influx. For intracellular cytokine staining, splenocytes were stimulated with $50 \mu \mathrm{g} / \mathrm{mL}$ PMA and $1 \mu \mathrm{M}$ ionomycin for 4 hours. Cells were incubated with brefeldin $\mathrm{A}$ for the final 3 hours of stimulation. After stimulation, cells were fixed and permeabilized with a Transcription Factor Staining Kit (eBioscience) and stained using anti-IL-4, anti-IFN- $\gamma$, or recombinant mouse IL-21R Fc chimera followed by a phycoerythrin-labeled (PE-labeled), affinity-purified $\mathrm{F}\left(\mathrm{ab}^{\prime}\right)_{2}$ fragment of goat anti-human Fc $\gamma$. For detection of phosphorylated antigens, splenocytes were fixed in BD Fixation Buffer for 20 minutes at room temperature (RT). Next, cells were washed and permeabilized in $90 \%$ methanol for 30 minutes at $-20^{\circ} \mathrm{C}$ and then incubated with antibody for 45 minutes at RT. The antibodies used are described in Supplemental Table 3. Data were acquired on a BD FACSCanto and analyzed with FlowJo (Tree Star) software.

Cell cultures and transfections. Ramos cells were grown in Iscove's DMEM with $10 \%$ FBS and $100 \mathrm{U} / \mathrm{mL}$ penicillin/streptomycin. Cell lines were monitored with the MycoAlert Mycoplasma Detection Kit and cultured under mycoplasma-free conditions. 293T cells were grown in DMEM with $10 \%$ FBS and $100 \mathrm{U} / \mathrm{mL}$ penicillin/streptomycin and transfected using the Mirus Transfection Kit with SREBP2, HA-IRF8(WT), or HA-IRF8(S164A) constructs. For murine cultures, single-cell suspensions from pooled spleens were enriched for B cells using biotinylated anti-CD23 and streptavidin microbeads. CD $23^{+} \mathrm{B}$ cells were cultured in RPMI 1640 medium with 10\% FBS, $100 \mathrm{U} / \mathrm{mL}$ penicillin/streptomycin, $1 \times$ nonessential amino acids, $2 \mathrm{mM} \mathrm{L-glu-}$ tamine, $25 \mathrm{mM}$ HEPES (pH 7.2-7.6), and $50 \mu \mathrm{M} \beta$-mercaptoethanol, followed by stimulation with $5 \mu \mathrm{g} / \mathrm{mL} \mathrm{F}\left(\mathrm{ab}^{\prime}\right) 2$ anti-mouse IgM and 5 $\mu \mathrm{g} / \mathrm{mL}$ anti-mouse CD40 with or without $50 \mathrm{ng} / \mathrm{mL}$ IL-21.

RT-qPCR and ChIP assays. Total RNA was isolated using the RNeasy Plus Mini Kit (QIAGEN). cDNAs were prepared using the iScript cDNA synthesis kit. RT-qPCR was performed using the iTaq Universal SYBR Green Supermix. Gene expression was calculated using the $\Delta \Delta \mathrm{Ct}$ method and normalized to Ppia. For ChIP assays, cells were harvested and chromatin extracts prepared using the truChIP Chromatin Shearing Reagent Kit (Covaris). One hundred micrograms of the sonicated DNA-protein complexes was used for immunoprecipitations with anti-SREBP2, anti-IRF8, anti-PU.1, or normal anti-rabbit
Ig control antibodies. DNA purified from the immunoprecipitates and inputs was analyzed by RT-qPCR. All primers used are listed in Supplemental Table 2.

Immunoblot analysis, kinase activity assays, and oligonucleotide precipitation assays. Extracts were prepared as previously described (28). The antibodies used for immunoblotting are detailed in Supplemental Table 3. The complete unedited blots are shown in the supplemental material. For ROCK kinase activity assays, ROCK1 or ROCK2 was immunoprecipitated from nuclear extracts using anti-ROCK1 or antiROCK2 antibodies as described previously (28). ROCK-mediated phosphorylation of IRF8 was assessed by in vitro ROCK2 kinase assays using recombinant active ROCK2 and immunoprecipitated IRF8 protein lacking the DNA-binding domain (IRF8 $\Delta 1-150)$. Briefly, immunoprecipitated IRF8 $\Delta 1-150$ was incubated with $50 \mathrm{ng}$ purified active ROCK2 in kinase buffer (25 mM Tris, pH 7.5, $10 \mathrm{mM} \mathrm{MgCl}, 5$ $\mathrm{mM} \beta$-glycerolphosphate, $0.1 \mathrm{mM} \mathrm{Na}_{3} \mathrm{VO}_{4}$, and $2 \mathrm{mM} \mathrm{DTT}$ ) containing $1 \mu \mathrm{M}$ ATP for 30 minutes at $30^{\circ} \mathrm{C}$. The kinase reactions were terminated by adding SDS-PAGE sample buffer and resolved on a $10 \%$ SDS-PAGE gel followed by detection of phosphorylated products using an antibody specific for phosphorylated serine residues. ONP assays were conducted as described previously (28). The oligonucleotide sequences are listed in Supplemental Table 2. RHOA activation assays were performed using the RhoA G-LISA Activation Assay Kit (Cytoskeleton Inc.).

ELISAs, ELISPOT assays, and total cholesterol assays. For the total Ig ELISA, plates were coated with $10 \mu \mathrm{g} / \mathrm{mL}$ goat anti-mouse Ig at $4^{\circ} \mathrm{C}$ overnight and blocked in 1\% BSA in PBS at RT for 1 hour. For the NP-specific Ig ELISA, plates were coated with $50 \mu \mathrm{g} / \mathrm{mL}$ NP-BSA conjugated at the appropriate ratio at $4^{\circ} \mathrm{C}$ overnight and blocked in 2\% BSA in PBS at RT for 1 hour. Sera were diluted at various ratios and incubated on coated plates at $25^{\circ} \mathrm{C}$ for 2 hours. Plates were then incubated with either alkaline phosphate-labeled or HRP-labeled goat anti-mouse IgM, IgG1, IgG2c, IgG3, or IgA Fc antibody for 1 hour before development. For the total IgE Ig ELISA, the manufacturer's protocol was followed. For ELISPOT assays, plates were coated overnight at $4^{\circ} \mathrm{C}$ with $100 \mu \mathrm{g} / \mathrm{mL}$ goat anti-mouse Ig for detection of total Ig ASCs or $50 \mu \mathrm{g} / \mathrm{mL}$ NP-BSA conjugated at the appropriate ratio $\left(\mathrm{NP}_{>30}\right.$ and $\left.\mathrm{NP}_{<8}\right)$ for detection of NP-specific Ig ASCs. Nonspecific binding was blocked with 3\% BSA and $5 \% \mathrm{FBS}$ in PBS, and samples were incubated at $37^{\circ} \mathrm{C}$ for 2 hours. Antibodies conjugated to biotin (goat anti-mouse IgG or goat antimouse $\operatorname{IgM}$ ) were added and incubated overnight at $37^{\circ} \mathrm{C}$ followed by streptavidin-alkaline-phosphate and detection using 5-bromo-4chloro-3-indolyl phosphate (BCIP). For total cholesterol assays, cell extracts from sorted cell populations were prepared with $1 \%$ $\mathrm{NP}_{40}$-based lysis buffer. Assays were performed using a total cholesterol assay kit (Cell Biolabs). Analyses were calculated by pooling single replicates from 2 independent experiments.

Histology and immunofluorescence staining. For H\&E staining, spleens were fixed in formalin for 4 hours and embedded in paraffin. For immunofluorescence staining, half the spleens were embedded in OCT and frozen in 2-methylbutane surrounded by dry ice. Frozen blocks were cut into $9-\mu \mathrm{m}$ sections with cryotome and stored at $-80^{\circ} \mathrm{C}$. Upon thawing, sections were allowed to dry at RT, fixed in cold acetone for 15 minutes, washed in PBS, and incubated in a blocking solution ( $0.3 \%$ BSA in PBS) for 15 minutes to minimize nonspecific binding. Sections were then incubated with antibodies for 25 minutes 
to 1 hour at RT. Signal from the primary antibodies was detected by staining with streptavidin-Alexa 594, goat anti-rat-BV-horizon, or donkey anti-rabbit-Alexa 488. Images were acquired with a Nikon Eclipse microscope and analyzed with Image (NIH).

In vitro cell conjugate assays. In vitro cell conjugate assays were conducted as described previously (6). Briefly, OT-II T cells were activated with anti-CD3 and anti-CD28 antibodies and cultured for 4 days with $10 \mu \mathrm{g} / \mathrm{mL}$ recombinant IL-2. Activated $\mathrm{T}$ cells were labeled with PerCP-conjugated anti-CD3. B cells were activated for 2 days with $0.4 \mu \mathrm{g} / \mathrm{mL}$ LPS and labeled with Cell Trace Violet. The labeled B cells $\left(5 \times 10^{5}\right.$ cells/well $)$ were plated in a 96-well plate and pulsed for 30 minutes at $37^{\circ} \mathrm{C}$ with increasing doses of OVA peptide (amino acids 323-339). Activated OT-II T cells were then added (5 $\times$ $10^{5}$ cells/well), and plates were centrifuged at RT for 5 minutes. The cell pellets were incubated for 30 minutes at $37^{\circ} \mathrm{C}$ and immediately acquired on a BD FACS Canto.

$\mathrm{J}_{\mathrm{H}} 4$ sequencing. $\mathrm{J}_{\mathrm{H}} 4$ sequencing was performed as described previously (57). In brief, an intronic region $3^{\prime}$ to the JH4 exon of IgH was PCR amplified from genomic DNA extracted from sorted GC B cells and follicular B cells from WT and C $\gamma 1-$ Rock2 mice. PCR products were cloned into the pCR4-Bunt-TOPO vector and sequenced with GeneWiz. The obtained $\mathrm{J}_{\mathrm{H}} 4$ intronic sequences were aligned to the $\mathrm{mm} 9$ assembly of the mouse genomic sequence. The primers used for $\mathrm{J}_{\mathrm{H}} 4$ sequencing are listed in Supplemental Table 2.

RNA-Seq analysis. The quality of all RNA and library preparations was evaluated with BioAnalyzer 2100 (Agilent Technologies). Sequencing libraries were sequenced by the Epigenomics Core Facility at Weill Cornell Medicine using a HiSeq 2500, 50-bp paired-end reads at a depth of approximately 22 to 30 million reads per sample. Read quality was assessed and adaptors trimmed using FASTP (58). Reads were then mapped to the mouse genome ( $\mathrm{mm} 10)$, and reads in exons were counted against Gencode v27 with STAR2.6 Aligner (59). Differential gene expression analysis was performed in R using edgeR 3.24.3. Genes with low expression levels ( $<2$ counts per million in at least 1 group) were filtered from all downstream analyses. Replica-associated batch correction was performed by directly incorporating a batch-specific term into a linear model. Differential expression was estimated using a quasi-likelihood framework. The Benhamini-Hochberg FDR procedure was used to correct for multiple testing. Genes with an unadjusted $P$ value of less than 0.01 were considered differentially expressed. Downstream analyses were performed in $\mathrm{R}$ using a visualization platform build with Shiny developed by bioinformaticians at the David Z. Rosensweig Genomics Research Center at the HSS.

GSEA was performed using GSEA software (Broad Institute) (60). Genes were ranked by the difference of log-transformed counts per million for contrasted conditions. The Molecular Signatures DataBase, version 62 (Broad Institute) was used as a source of gene sets with defined functional relevance. Gene sets ranging between 15 and 1000 genes were included in the analysis. Nominal $P$ values were FDR corrected, and gene sets with an FDR below 0.05 were used to create GSEA enrichment plots.
ATAC-Seq analysis. The nuclei of sorted WT and CD23-Rock2 GC B cells were prepared by incubation of cells with nuclear preparation buffer (0.30 M sucrose, $10 \mathrm{mM}$ Tris, pH 7.5, $60 \mathrm{mM} \mathrm{KCl,} 15 \mathrm{mM}$ $\mathrm{NaCl}, 5 \mathrm{mM} \mathrm{MgCl}$, $0.1 \mathrm{mM}$ EGTA, 0.1\% NP40, $0.15 \mathrm{mM}$ spermine, $0.5 \mathrm{mM}$ spermidine, and $2 \mathrm{mM} 6 \mathrm{AA}$ ) (61). Libraries were prepared as previously described (62). Paired-end, 50-bp sequences were generated from samples with an Illumina HiSeq 2500 and, following adapter trimming with FastP, were aligned against the mouse genome (mm10) using bowtie2 with --local -q -p options. Peaks were called using MACS2 with --macs2 callpeak -f BAMPE --nomodel --shift -100 --extsize 200 -B --SPMR -g \$GENOMESIZE -q 0.01 options. De novo transcription factor motif analysis was performed with HOMER on ATAC-Seq peaks found within $10 \mathrm{~kb}$ upstream of the transcription start site of genes differentially expressed $(P<0.01$, FC $>2$ ) in the RNA-Seq between WT and CD23-Rock2 GC B cells.

Statistics. $P$ values were calculated using an unpaired, 2-tailed $t$ test for 2-group comparisons and by 1-way ANOVA followed by either Tukey's or Dunnet's test for multiple group comparisons. $P$ values of less than 0.05 were considered significant. Statistical analyses were performed using GraphPad Prism 7 (GraphPad Software).

The RNA-Seq and ATAC-Seq data were deposited in the NCBI's Gene Expression Omnibus (GEO) database (GEO GSE141328).

Study approval. All experiments were performed according to the protocols approved by the ACUC of the HSS, Weill Cornell Medicine.

\section{Author contributions}

ER and ABP designed the studies. ER, TP, DFC, CY, SG, and MM performed the experiments. JKL provided input on the manuscript content and developed experimental models. YC performed bioinformatics analyses. ER and ABP interpreted the data and wrote the manuscript.

\section{Acknowledgments}

We thank J. Chaudhuri (Memorial Sloan Kettering Cancer Center [MSKCC], New York, New York, USA) for the CD23-Cre mice. We also thank members of the HSS Research Institute for thoughtful discussions and reagents. This work was supported by the NIH (AR064883 and AR070146, to ABP, and a T32 Rheumatology Training Grant, to ER); the Peter Jay Sharp Foundation; the Tow Foundation, which provided support for the David Z. Rosensweig Genomics Research Center; the Giammaria Giuliani Foundation; the Ambrose Monell Foundation; and the Barbara Volcker Center for the Michael D. Lockshin Fellowship (to MM). Technical support as provided by the Epigenomics Core and the Flow Cytometry Core Facility of Weill Cornell Medical College and from the Office of the Director of the NIH under award number S10OD019986 (to the HSS).

Address correspondence to: Alessandra B. Pernis, Autoimmunity and Inflammation Program, Hospital for Special Surgery, 535 East 70th Street, New York, New York 10021, USA. Phone: 212.206.1612; Email:pernisa@hss.edu.
1. Shlomchik MJ, Luo W, Weisel F. Linking signaling and selection in the germinal center. Immunol Rev. 2019;288(1):49-63.

2. Lu E, Cyster JG. G-protein coupled receptors and ligands that organize humoral immune responses.
Immunol Rev. 2019;289(1):158-172.

3. Mesin L, Ersching J, Victora GD. Germinal center B cell dynamics. Immunity. 2016;45(3):471-482.

4. De Silva NS, Klein U. Dynamics of B cells in germinal centres. Nat Rev Immunol.
2015;15(3):137-148.

5. Zotos D, et al. IL-21 regulates germinal center $B$ cell differentiation and proliferation through a B cell-intrinsic mechanism. J Exp Med. 2010;207(2):365-378. 
6. $\mathrm{Xu} \mathrm{H}$, et al. Regulation of bifurcating $\mathrm{B}$ cell trajectories by mutual antagonism between transcription factors IRF4 and IRF8. Nat Immunol. 2015;16(12):1274-1281.

7. Carotta S, et al. The transcription factors IRF8 and PU.1 negatively regulate plasma cell differentiation. J Exp Med. 2014;211(11):2169-2181.

8. Feng J, Wang H, Shin DM, Masiuk M, Qi CF, Morse HC. IFN regulatory factor 8 restricts the size of the marginal zone and follicular B cell pools. JImmunol. 2011;186(3):1458-1466.

9. Lee $\mathrm{CH}$, et al. Regulation of the germinal center gene program by interferon (IFN) regulatory factor 8/IFN consensus sequence-binding protein. JExp Med. 2006;203(1):63-72.

10. Wang H, et al. Transcription factors IRF8 and PU.1 are required for follicular B cell development and BCL6-driven germinal center responses. Proc Natl Acad Sci USA. 2019;116(19):9511-9520.

11. Dominguez-Sola D, et al. The FOXO1 transcription factor instructs the germinal center dark zone program. Immunity. 2015;43(6):1064-1074.

12. Sander S, et al. PI3 kinase and FOXO1 transcription factor activity differentially control B cells in the germinal center light and dark zones. Immunity. 2015;43(6):1075-1086.

13. Luo W, Weisel F, Shlomchik MJ. B cell receptor and CD40 signaling are rewired for synergistic induction of the c-Myc transcription factor in germinal center B cells. Immunity. 2018;48(2):313-326.e5.

14. Lu E, Wolfreys FD, Muppidi JR, Xu Y, Cyster JG. S-geranylgeranyl-L-glutathione is a ligand for human B cell-confinement receptor P2RY8. Nature. 2019;567(7747):244-248.

15. Gatto D, Paus D, Basten A, Mackay CR, Brink R. Guidance of B cells by the orphan $G$ proteincoupled receptor EBI2 shapes humoral immune responses. Immunity. 2009;31(2):259-269.

16. Green JA, et al. The sphingosine 1-phosphate receptor $\mathrm{S}_{1} \mathrm{P}_{2}$ maintains the homeostasis of germinal center $B$ cells and promotes niche confinement. Nat Immunol. 2011;12(7):672-680.

17. Pereira JP, Kelly LM, Xu Y, Cyster JG. EBI2 mediates $\mathrm{B}$ cell segregation between the outer and centre follicle. Nature. 2009;460(7259):1122-1126.

18. Boothby M, Rickert RC. Metabolic regulation of the immune humoral response. Immunity. 2017;46(5):743-755.

19. Jellusova J, et al. Gsk3 is a metabolic checkpoint regulator in B cells. Nat Immunol. 2017;18(3):303-312.

20. Ersching J, et al. Germinal center selection and affinity maturation require dynamic regulation of mTORC1 kinase. Immunity. 2017;46(6):1045-1058.e6.

21. Raybuck AL, et al. B cell-intrinsic mTORC1 promotes germinal center-defining transcription factor gene expression, somatic hypermutation, and memory B cell generation in Humoral Immunity. J Immunol. 2018;200(8):2627-2639.

22. Luo W, et al. The AKT kinase signaling network is rewired by PTEN to control proximal BCR signaling in germinal center B cells. Nat Immunol. 2019;20(6):736-746.

23. Julian L, Olson MF. Rho-associated coiled-coil containing kinases (ROCK): structure, regulation, and functions. Small GTPases. 2014;5:e29846.

24. Pernis AB, Ricker E, Weng CH, Rozo C, Yi W. Rho kinases in autoimmune diseases. Anпи Rev Med. 2016;67:355-374.

25. Zhou Q, Gensch C, Liao JK. Rho-associated coiled-coil-forming kinases (ROCKs): potential targets for the treatment of atherosclerosis and vascular disease. Trends Pharmacol Sci. 2011;32(3):167-173.

26. Muppidi JR, et al. Loss of signalling via Ga13 in germinal centre B-cell-derived lymphoma. Nature. 2014;516(7530):254-258.

27. Lepley D, Paik JH, Hla T, Ferrer F. The G proteincoupled receptor S1P2 regulates Rho/Rho kinase pathway to inhibit tumor cell migration. Cancer Res. 2005;65(9):3788-3795.

28. Biswas PS, et al. Phosphorylation of IRF 4 by ROCK2 regulates IL-17 and IL-21 production and the development of autoimmunity in mice. J Clin Invest. 2010;120(9):3280-3295.

29. Zanin-Zhorov A, et al. Selective oral ROCK2 inhibitor down-regulates IL-21 and IL-17 secretion in human T cells via STAT3-dependent mechanism. Proc Natl Acad Sci USA. 2014;111(47):16814-16819.

30. Weiss JM, et al. ROCK2 signaling is required to induce a subset of $\mathrm{T}$ follicular helper cells through opposing effects on STATs in autoimmune settings. Sci Signal. 2016;9(437):ra73.

31. Goenka R, et al. Local BLyS production by $\mathrm{T}$ follicular cells mediates retention of high affinity $\mathrm{B}$ cells during affinity maturation. J Exp Med. 2014;211(1):45-56.

32. Basso K, et al. Tracking CD40 signaling during germinal center development. Blood. 2004;104(13):4088-4096.

33. Saito $\mathrm{M}$, et al. A signaling pathway mediating downregulation of BCL6 in germinal center B cells is blocked by BCL6 gene alterations in B cell lymphoma. Cancer Cell. 2007;12(3):280-292.

34. Kwon K, et al. Instructive role of the transcription factor E2A in early B lymphopoiesis and germinal center B cell development. Immunity. 2008;28(6):751-762.

35. Rosado MM, Scarsella M, Cascioli S, Giorda E, Carsetti R. Evaluating B-cells: from bone marrow precursors to antibody-producing cells. Methods Mol Biol. 2013;1032:45-57.

36. Casola S, et al. Tracking germinal center B cells expressing germ-line immunoglobulin gamma1 transcripts by conditional gene targeting. Proc Natl Acad Sci USA . 2006;103(19):7396-7401.

37. Klein U, et al. Transcription factor IRF4 controls plasma cell differentiation and class-switch recombination. Nat Immunol. 2006;7(7):773-782.

38. Jolly CJ, Klix N, Neuberger MS. Rapid methods for the analysis of immunoglobulin gene hypermutation: application to transgenic and gene targeted mice. Nucleic Acids Res. 1997;25(10):1913-1919.

39. Jiang $X$, et al. HGAL, a germinal center specific protein, decreases lymphoma cell motility by modulation of the RhoA signaling pathway. Blood. 2010;116(24):5217-5227.

40. Wang Y, et al. Germinal-center development of memory B cells driven by IL-9 from follicular helper T cells. Nat Immunol. 2017;18(8):921-930.

41. Manning BD, Toker A. AKT/PKB signaling: navigating the network. Cell. 2017;169(3):381-405.

42. Li Z, et al. Regulation of PTEN by Rho small GTPases. Nat Cell Biol. 2005;7(4):399-404. 43. Alleaume-Butaux A, et al. Double-edge sword of sustained ROCK activation in prion diseases through neuritogenesis defects and prion accumulation. PLoS Pathog. 2015;11(8):e1005073.

44. Albright AR, Kabat J, Li M, Raso F, Reboldi A, Muppidi JR. TGF $\beta$ signaling in germinal center B cells promotes the transition from light zone to dark zone. J Exp Med. 2019;216(11):2531-2545.

45. Sage PT, Sharpe AH. T follicular regulatory cells. Immunol Rev. 2016;271(1):246-259.

46. Shao W, Espenshade PJ. Expanding roles for SREBP in metabolism. Cell Metab. 2012;16(4):414-419.

47. Kim YC, et al. Liver ChIP-seq analysis in FGF19-treated mice reveals SHP as a global transcriptional partner of SREBP-2. Genome Biol. 2015;16:268.

48. Hodge RG, Ridley AJ. Regulating Rho GTPases and their regulators. Nat Rev Mol Cell Biol. 2016;17(8):496-510.

49. Förster R, et al. CCR7 coordinates the primary immune response by establishing functional microenvironments in secondary lymphoid organs. Cell. 1999;99(1):23-33.

50. Flynn R, et al. Targeted Rho-associated kinase 2 inhibition suppresses murine and human chronic GVHD through a Stat3-dependent mechanism. Blood. 2016;127(17):2144-2154.

51. Reboldi A, Dang E. Cholesterol metabolism in innate and adaptive response. F1000Res. 2018;7:F1000 Faculty Rev-1647.

52. Chen L, et al. SYK inhibition modulates distinct PI3K/AKT- dependent survival pathways and cholesterol biosynthesis in diffuse large B cell lymphomas. Cancer Cell. 2013;23(6):826-838.

53. Chmielewski S, Piaszyk-Borychowska A, Wesoly J, Bluyssen HA. STAT1 and IRF8 in vascular inflammation and cardiovascular disease: diagnostic and therapeutic potential. Int Rev Immunol. 2016;35(5):434-454.

54. Masuda T, et al. IRF8 is a critical transcription factor for transforming microglia into a reactive phenotype. Cell Rep. 2012;1(4):334-340.

55. Xia Y, et al. The mevalonate pathway is a druggable target for vaccine adjuvant discovery. Cell. 2018;175(4):1059-1073.e21.

56 . Okamoto R, et al. FHL2 prevents cardiac hypertrophy in mice with cardiac-specific deletion of ROCK2. FASEB J. 2013;27(4):1439-1449.

57. Nicolas L, et al. Cutting Edge: ATM influences germinal center integrity. J Immunol. 2019;202(11):3137-3142.

58. Chen S, Zhou Y, Chen Y, Gu J. fastp: an ultra-fast all-in-one FASTQ preprocessor. Bioinformatics. 2018;34(17):i884-i890.

59. Dobin A, et al. STAR: ultrafast universal RNA-seq aligner. Bioinformatics. 2013;29(1):15-21.

60. Subramanian A, et al. Gene set enrichment analysis: a knowledge-based approach for interpreting genome-wide expression profiles. Proc Natl Acad Sci USA. 2005;102(43):15545-15550.

61. Minnich M, et al. Multifunctional role of the transcription factor Blimp-1 in coordinating plasma cell differentiation. Nat Immunol. 2016;17(3):331-343.

62. Buenrostro JD, Giresi PG, Zaba LC, Chang HY, Greenleaf WJ. Transposition of native chromatin for fast and sensitive epigenomic profiling of open chromatin, DNA-binding proteins and nucleosome position. Nat Methods. 2013;10(12):1213-1218. 